\title{
A light-cone wavefunction approach to open heavy flavor dynamics in QCD matter
}

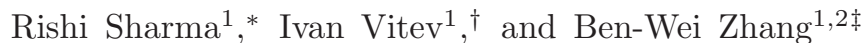 \\ 1 Los Alamos National Laboratory, Theoretical Division, Los Alamos, NM 87545, USA and \\ ${ }^{2}$ Key Laboratory of Quark \& Lepton Physics (Huazhong Normal University), Ministry of Education, China
}

\begin{abstract}
We calculate the lowest order charm and beauty parton distribution functions in and fragmentation functions into $D$ and $B$ mesons using the operator definitions of factorized perturbative QCD. In the vacuum, we find the leading corrections that arise from the structure of the final-state hadrons. Quark-antiquark potentials extracted from the lattice are employed to demonstrate the existence of open heavy flavor bound-state solutions in the QGP in the vicinity of the critical temperature. We provide first results for the in-medium modification of the heavy quark distribution and decay probabilities in a co-moving plasma. In an improved perturbative QCD description of heavy flavor dynamics in the thermal medium, we combine $D$ and $B$ meson formation and dissociation with parton-level charm and beauty quark quenching to obtain predictions for the heavy meson and non-photonic electron suppression in $\mathrm{Cu}+\mathrm{Cu}$ and $\mathrm{Pb}+\mathrm{Pb}$ collisions at RHIC and the LHC, respectively.
\end{abstract}

PACS numbers: 12.38.Bx; 12.39.Ki; 13.87.Fh; 24.85.+p

\section{INTRODUCTION}

The early production of heavy quarks makes them some of the most important probes of the quark-gluon plasma (QGP) created in ultra-relativistic collisions of heavy nuclei [1]. Precise and direct measurements of the multiplicities and differential distributions of $D$ and $B$ hadrons will soon become available with the vertex detector upgrades at the Relativistic Heavy Ion Collider (RHIC) and at the Large Hadron Collider (LHC). Such experimental advances will allow to quantitatively address the key observable in high temperature Quantum Chromo-Dynamics (QCD) - the apparent modification of particle production by energetic partons traversing a region of hot nuclear matter [2, 3, 4] - for heavy quark jets. In the framework of perturbative QCD (pQCD), studies in this direction have focused so far exclusively, with varying degree of sophistication, on the loss of the leading parton or particle energy via radiative and collisional processes [5, 6] (commonly known as jet quenching). This energy loss leads to a suppression of the observed number of energetic particles in heavy ion $(\mathrm{A}+\mathrm{B})$ collisions relative to their number in proton-proton $(\mathrm{p}+\mathrm{p}$, or $\mathrm{N}+\mathrm{N})$ reactions, scaled by the number of binary collisions.

It is well known that $c$ and $b$ quark partonic level energy loss in the QGP is not sufficient to explain the

\footnotetext{
*Electronic address: rishi@lanl.gov

$\dagger$ Electronic address: ivitev@lanl.gov

${ }^{\ddagger}$ Electronic address: bzhang@lanl.gov
}

large suppression of non-photonic $e^{+}+e^{-}$measured at RHIC [6]. These non-photonic electrons predominantly arise from the semi-leptonic decays of $D$ and $B$ mesons that are usually considered to be formed by heavy quark fragmentation outside of the medium. However, due to their small formation times, it is possible that $D$ and $B$ mesons could form inside the medium and subsequently dissociate [7]. For this reason, effective energy loss via meson dissociation in the QGP [7, 8, 9] has also been studied in recent publications. Heavy meson dissociation approaches have been quite successful phenomenologically 7], but the question of the possible thermal modifications to $D$ and $B$ hadrons has remained open. Even though such modifications will affect fragmentation only if the time scale for their onset is smaller than the inverse virtuality in the parton decay, it is surprising that to date there has been no theoretical evaluation of the quark distribution (PDF) and fragmentation (FF) functions at $T \neq 0$.

Heavy flavor provides the ideal testing ground for our first quantitative study of such many-body QCD effects because the lowest-lying Fock state in the light-cone wavefunction expansion for $D$ and $B$ mesons [10, 11] can be related to the hadron rest-frame solution of the corresponding Dirac equation [12, 13]. Furthermore, potential model calculations can be generalized up to temperatures of order few $T_{c}$ 14 using lattice QCD input 15. In this paper we provide the first calculation of the thermal modification of PDFs and FFs for open heavy flavor. As we will demonstrate below, even in equilibrium with a comoving plasma heavy-light quark bound-state solutions 
of the Dirac equation persist to temperatures $\sim 1.6 T_{c}$. This provides strong motivation to take the existing studies of open heavy flavor dynamics in dense QCD matter a step further by combining the partonic and hadronic aspects of the observed high- $p_{T}$ cross section attenuation.

For the realistic case of out-of-equilibrium fast jet propagation in the thermal medium we combine the partonic level quenching with $D$ and $B$ meson dissociation in the medium to compute the spectrum of $D$ and $B$ mesons prior to their weak decay. We compare the relative importance of charm and beauty quark energy loss versus meson dissociation as a function of transverse momentum. We also show results for the non-photonic electron suppression in central $\mathrm{Au}+\mathrm{Au}$ and $\mathrm{Cu}+\mathrm{Cu}$ reactions at $\sqrt{s_{N N}}=200 \mathrm{GeV}$ and central $\mathrm{Pb}+\mathrm{Pb}$ collisions at $\sqrt{s_{N N}}=5.5 \mathrm{TeV}$. We discuss the prospects of testing the pQCD theory by upcoming open heavy flavor measurements at RHIC and the LHC.

Our paper is organized as follows. In Section [I] we present a detailed baseline calculation of charm and beauty quark production, inclusive of the known cold nuclear matter effects, and expose the limitations of the purely partonic approach to their final-state dynamics in the QGP. In Section [II we express the parton distribution and decay probabilities of heavy quarks in hadrons in terms of the light-cone wavefunctions and calculate the $c$ and $b$ quark FFs. In Section IV we evaluate the heavy meson wavefunctions in the vacuum and in the vicinity of the QCD phase transition and present numerical results for the corresponding distribution and decay probabilities. A complete calculation that includes $D$ and $B$ meson dissociation and $c$ and $b$ quark quenching, relevant to current and future heavy flavor/non-photonic electron measurements, is given in Section $\mathrm{V}$. A summary and conclusions are presented in Section VI. Background information and some of the technical aspects of this work are given in the Appendix. Appendix $\AA$ describes notation and quantization of parton fields. In Appendix B we elucidate the relation between the instant and light-cone forms of a heavy meson wavefunction. Demonstration of the smallness of the thermal light quark pick-up correction to the heavy quark FFs is given in Appendix C] Appendix D presents an analytic solution for the evolution of the heavy quark and meson system for time-averaged fragmentation and dissociation rates.

\section{A TIMELESS LIMIT}

To correctly assess the discrepancy between the expected heavy quark quenching and the measured nonphotonic electron suppression [16, 17] improved treatment of cold nuclear matter effects is necessary. A previous study of $D$ and $B$ meson production in $\mathrm{p}+\mathrm{A}$ reactions [18] indicated that these will affect the nuclear modification ratio $\left(R_{A B}\right)$ for energetic particles [4]. This ratio is defined as follows:

$$
R_{A B}(\mathbf{p})=\frac{d \sigma_{A B}}{d y d^{2} \mathbf{p}} /\left(N_{\text {bin. }}^{A B}\right) \frac{d \sigma_{N N}}{d y d^{2} \mathbf{p}},
$$

and is used as one of the main observables to characterize the properties of the system created in heavy ion reactions. In Eq. (10), $\sigma_{A B}$ refers to the production crosssection in collisions between two heavy ions A and B, $\sigma_{N N}$ refers to the cross-section in collisions between two nucleons $\mathrm{N}$, and $N_{\text {bin. }}^{A B}$ refers to the number of binary $\mathrm{N}+\mathrm{N}$ collisions in an $\mathrm{A}+\mathrm{B}$ reaction. $N_{\text {bin }}^{A B}$ is determined by using a Glauber model [19]. It is, therefore, important to establish the role of shadowing, Cronin effect and cold nuclear matter energy loss in the analysis of open heavy flavor suppression. Note that in our notation $\mathbf{p}, \mathbf{k} \ldots$ are $2 \mathrm{D}$ transverse vectors and, for example, $p_{T}=|\mathbf{p}|$.

At the partonic level, the cross sections per elementary nucleon-nucleon collision, including the ones for charm and beauty quarks, can be calculated as follows:

$$
\begin{aligned}
& \left(N_{\text {bin. }}^{A B}\right)^{-1} \frac{d \sigma_{A B}^{q, g}}{d y d^{2} \mathbf{p}}=K \sum_{a b c d} \int d y_{d} \int d^{2} \mathbf{k}_{a} d^{2} \mathbf{k}_{b} \frac{f\left(k_{T a}\right) f\left(k_{T b}\right)}{\left|J\left(k_{T a}, k_{T b}\right)\right|} \\
& \times \frac{\alpha_{s}^{2}\left(\mu_{r}\right)}{2 S}\left|\bar{M}_{a b \rightarrow c d}\right|^{2} \frac{\phi_{a / N}\left(\frac{\tilde{x}_{a}}{1-\epsilon_{a}}, \mu_{f}\right) \phi_{b / N}\left(\frac{\tilde{x}_{b}}{1-\epsilon_{b}}, \mu_{f}\right)}{\tilde{x}_{a} \tilde{x}_{b}} .
\end{aligned}
$$

In Eq. (2) $\mathbf{k}_{a}, \mathbf{k}_{b}$ refer to the transverse momenta of the two partons $a, b$, respectively, that participate in the hard collision and $x_{a}, x_{b}$ is the fraction of the light cone momentum of the nucleon $\mathrm{N}$ carried by these. $M_{a b \rightarrow c d}$ denotes the matrix element of the hard collision of $a$ and $b$ to give rise to partons $c$ and $d . \quad \phi(x, \mu)$ refers to the parton distribution functions that give the probability to find a parton with light cone momentum fraction $x$ of the nucleon light cone momentum. We set the factorization and renormalization scales to $\mu_{f}=\mu_{r}=m_{T}=\left(m_{q, g}^{2}+\right.$ $\left.p_{T}^{2}\right)^{1 / 2}$ and the Jacobian $J$ reads:

$$
J_{x_{a}, x_{b}}\left(k_{T a}, k_{T b}\right)=\frac{S}{2}\left(1-\frac{k_{T a}^{2} k_{T b}^{2}}{x_{a}^{2} S x_{b}^{2} S}\right),
$$

subject to the hard scattering constraint $k_{T a, b}<x_{a, b} \sqrt{S}$. We have generalized the pQCD collinear factorization approach to heavy flavor production [18, 20] to account for the non-zero parton transverse momentum distribution $f\left(k_{T a, b}\right)$ in the hadron wavefunction with $\left\langle k_{T a, b}^{2}\right\rangle_{N N}=$ $0.9 \mathrm{GeV}^{2}$ 21]. The phenomenological next-to-leading order $K$ factor in Eq. (2) cancels in the observable $R_{A B}\left(p_{T}\right)$, Eq. (11). Finally, we note that the momentum fraction $x$ that appears in Eq. (2) is modified due to many-body QCD scattering effects that we discuss below.

Cronin effect. In $\mathrm{p}+\mathrm{A}$ and $\mathrm{A}+\mathrm{A}$ reactions the Cronin effect can be taken into account in the $\mathbf{p}$-differential cross sections by including the $\mathbf{k}$ broadening of incoming partons that arises from initial-state scattering [22, 23]. This transverse momentum broadening in heavy ion collisions is simplest to evaluate if $f\left(k_{T a, b}\right)$ is of a normalized Gaussian form due to the additive dispersion property: The 
width of the transverse distribution function $f$ grows relative to its value in $\mathrm{N}+\mathrm{N}$ collisions as follows:

$$
\begin{aligned}
& \left\langle k_{T a, b}^{2}\right\rangle_{A B}=\left\langle k_{T a, b}^{2}\right\rangle_{N N}+\left\langle k_{T a, b}^{2}\right\rangle_{I S}, \\
& \left\langle k_{T a, b}^{2}\right\rangle_{I S}=\left\langle\frac{2 \mu^{2} L}{\lambda_{q, g}}\right\rangle \xi
\end{aligned}
$$

Specifically, in Eq. (44) $\mu^{2}=0.12 \mathrm{GeV}^{2}, \quad \lambda_{g}=$ $\left(C_{F} / C_{A}\right) \lambda_{q}=1 \mathrm{fm}$ and $\xi \sim$ few is a numerical factor that accounts for the enhancement of the broadening coming from the power-law tails of the Moliere multiple scattering [22, 23].

Cold nuclear matter-energy loss. Initial-state multiple collisions also give rise to radiative energy loss that is sensitive to the quark or gluon mass. Theoretical derivation of the stopping power of large nuclei for partons was given in Ref. 24] and numerical calculations were carried out for the same squared transverse momentum transfers and parton mean free paths given above. If fast quarks or gluons lose a fraction $\epsilon_{a, b}=\frac{\Delta E_{a, b}^{\mathrm{rad}}}{E_{a, b}}$ of their energy prior to the hard scattering, to satisfy the same final-state kinematics they must initially carry a larger fraction of the colliding hadron momentum and, correspondingly, a larger value of $x$. This can be implemented in Eq. (2) as follows:

$$
\tilde{x}_{a} \rightarrow \frac{\tilde{x}_{a}}{1-\epsilon_{a}}, \quad \tilde{x}_{b} \rightarrow \frac{\tilde{x}_{b}}{1-\epsilon_{b}}, \quad \tilde{x}_{a, b} \leq 1,
$$

in the parton distribution functions $\phi_{a, b / N}\left(\tilde{x}_{a, b}, \mu_{f}\right)$. $\tilde{x}_{a, b}$ will be defined in terms of $x_{a, b}$ in a moment when we consider dynamical shadowing. We note that the theoretical calculation of cold nuclear matter energy loss is most easily performed in the target nucleus rest frame where the incident partons are typically of very high energy [24].

Dynamical shadowing. Last but not least, powersuppressed 25] resummed 26, 27] coherent final-state scattering of the struck partons that leads to shadowing in the observed cross sections is included via [18]:

$$
\begin{aligned}
& \tilde{x}_{a}=x_{a}\left(1+\frac{\xi_{d}^{2}\left(A^{1 / 3}-1\right)}{-\hat{t}+m_{d}^{2}}\right), \\
& \tilde{x}_{b}=x_{b}\left(1+\frac{\xi_{c}^{2}\left(A^{1 / 3}-1\right)}{-\hat{u}+m_{c}^{2}}\right) .
\end{aligned}
$$

In Eqs. (6) and (7) $\hat{t}, \hat{u}$ are the relevant Mandelstam variables at the partonic level and $m_{c}, m_{d}$ are the masses of the struck target partons. $A$ is the atomic number of the nucleus. A consistent picture of cold nuclear matter effects requires $\left(\xi^{2}\right)_{q, g} A^{1 / 3} \approx\left(2 \mu^{2} L / \lambda\right)_{q, g}$ in minimum bias reactions, which yields $\left(\xi^{2}\right)_{q} \approx 0.12 \mathrm{GeV}^{2}$ and $\left(\xi^{2}\right)_{g} \approx 0.27 \mathrm{GeV}^{2}$.

Our results, reported here, bring the treatment of cold nuclear matter effects for open heavy flavor production on par with their implementation in the study of light hadron and direct photon final states [28]. Linear dependence of cold nuclear matter effects on the mean nuclear thickness of the interaction region is implicit in our approach and reflects the actual theoretical findings.

We now turn to the final-state quark and gluon dynamics that is governed by the formation times of the plasma [30], $\tau_{0}$, and of the hadrons, $\tau_{\text {form }}$ 7]. Using the uncertainty principle, one can evaluate:

$$
\tau_{\text {form }} \simeq \frac{2 z(1-z) E_{T}}{\mathbf{k}^{2}+(1-z) m_{h}^{2}-z(1-z) m_{q}^{2}},
$$

which grows with underlying jet energy $\left(E_{T}\right)$, depends on the fractional momentum $z=E_{h} / E_{q, g}$ carried by the leading hadron and is inversely proportional to the square of its mass $\left(m_{h}\right)$. We take the formation time of the QGP to be $\tau_{0}=0.6 \mathrm{fm}$ and $\tau_{0}=0.3 \mathrm{fm}$ at RHIC and LHC, respectively, compatible with hydrodynamic studies [29]. Simulations of the early stages of the reaction [30] suggest that the transverse energy density of the partonic system builds through a short exponential growth. Therefore, for $\tau<\tau_{0}$ particles will be largely unaffected by the medium.

For $\tau \geq \tau_{0}$ the properties of the soft gluon-dominated matter, created in heavy ion reactions with multiplicity proportional to the participant density of the colliding nuclei:

$$
\frac{d N^{g}}{d y d^{2} \mathbf{x}}(\mathbf{x})=\kappa \frac{d N^{\text {part. }}}{d^{2} \mathbf{x}}(\mathbf{x})
$$

can be constrained by the experimentally measured charged particle pseudorapidity density [31]. Integrating over the position in the transverse plane $\mathrm{x}$ in Eq. (9) we can determine $\kappa$ from simple parton-hadron duality:

$$
\frac{d N^{g}}{d y}=\frac{3}{2}\left|\frac{d \eta}{d y}\right| \frac{d N^{c h}}{d \eta} .
$$

Here, $d N^{g} / d y$ refers to the gluon rapidity density, and $\eta=-\log (\tan (\theta / 2))$ is the pseudorapidity, where $\theta$ is the angle of the observed particle with respect to the beam axis. For example, in central $\mathrm{Cu}+\mathrm{Cu}$ and $\mathrm{Au}+\mathrm{Au}$ collisions at RHIC, at the average nucleon-nucleon collision energy, $\sqrt{s_{N N}}=200 \mathrm{GeV}, \frac{d N^{g}}{d y}=250$ and 1050, respectively, and in central $\mathrm{Pb}+\mathrm{Pb}$ collisions at the LHC at $\sqrt{s_{N N}}=5.5 \mathrm{TeV}$ one expects $\frac{d N^{g}}{d y} \simeq 2800$. The local time- and position-dependent temperature for an isentropic Bjorken expansion can be readily obtained:

$$
T(t, \mathbf{x})=\sqrt[3]{\frac{\pi^{2}}{16 \zeta(3)} \frac{1}{t} \frac{d N^{g}}{d y d^{2} \mathbf{x}}(\mathbf{x})} .
$$

In Eq. (11) we have replaced the proper time $\tau\left(t, x^{3}\right)=$ $\sqrt{t^{2}-\left(x^{3}\right)^{2}}$, where $x^{3}$ is the position coordinate along the axis of the collision, by the time $t$. This is a good approximation for $x^{3}$ small compared to $t$, meaning rapidities $y$ small compared to 1 . The Debye screening scale, $m_{D}=g T$, and the relevant gluon mean free path $\lambda_{g}=1 / \sigma^{g g} \rho$ with $\sigma^{g g}=(9 / 2) \pi \alpha_{s}^{2} / m_{D}^{2}$, that enter jet quenching calculations can then be evaluated. 


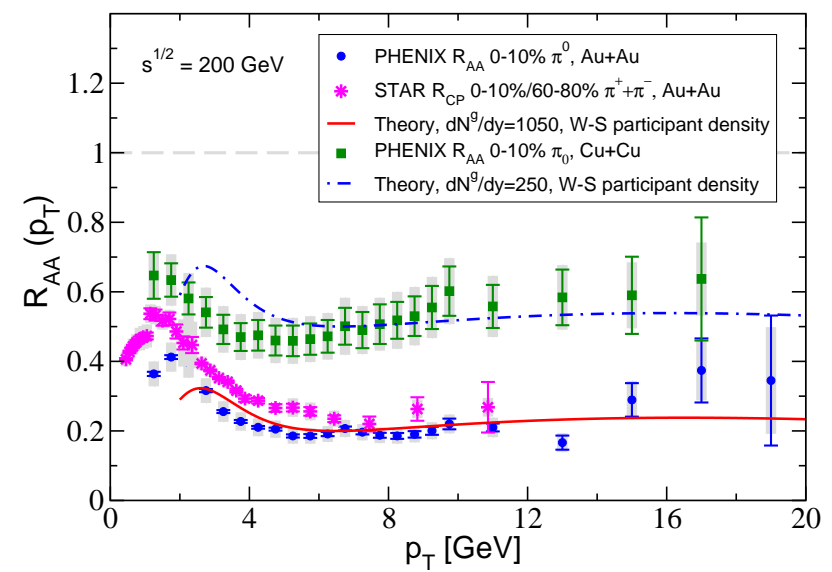

FIG. 1: Perturbative QCD calculation of the $\pi^{0}$ and $\pi^{+}+$ $\pi^{-}$quenching in central $\mathrm{Au}+\mathrm{Au}$ and $\mathrm{Cu}+\mathrm{Cu}$ collisions at RHIC at $\sqrt{s_{N N}}=200 \mathrm{GeV}$. Pion suppression data from PHENIX 33, 34] and STAR 35] is also shown.

In contrast to the soft gluons discussed above, large $Q^{2}$ parton processes follow a binary collision density distribution, $\propto \frac{d N^{b i n} .}{d^{2} \mathrm{x}}$, that is peaked near the center of the collision geometry. In Ref. [32] a complete and numerically intensive calculation of jet production and propagation through the plasma was carried out to confirm that the energy loss pattern is determined by the jets originating near the peak of the binary collision density. Therefore, we here focus only on these hard partons, use a running coupling constant at the radiation vertex and describe the strength of the interactions of the jet with the medium by an effective value $\alpha_{s}=0.28-0.32$. Any uncertainty associated with higher orders in opacity and peripheral jets will lead to somewhat larger values of $\alpha_{s}$. The energy loss calculation itself is performed in the framework of the GLV approach [4, 24] and the Landau-Pomeranchuk-Migdal destructive interference effect in QCD is fully accounted for. We evaluate the probability distribution $P(\epsilon)$ for the parton to lose a fraction of its energy loss $\epsilon=\sum_{i} \frac{\Delta E_{i}}{E}$ due to multiple gluon emission. The quenched quark or gluon spectrum is then readily obtained:

$$
\frac{d \sigma_{A B}^{q, g \text { Quench }}(\mathbf{p})}{d y d^{2} \mathbf{p}}=\int_{0}^{1} d \epsilon P(\epsilon) \frac{1}{(1-\epsilon)^{2}} \frac{d \sigma_{A B}^{q, g}\left(\frac{\mathbf{p}}{1-\epsilon}\right)}{d y d^{2} \mathbf{p}} .
$$

In Eq. (12) the factor $(1-\epsilon)^{-2}$ arises from the transverse phase space Jacobian $\left|d^{2} \mathbf{p} / d^{2} \mathbf{p}^{\text {Quench }}\right|$. Hadronization is performed using the appropriate vacuum fragmentation function $D_{h / q, g}\left(z, \mu_{f r}\right)$ as follows:

$$
\begin{aligned}
\frac{d \sigma_{A B}^{h}(\mathbf{p})}{d y d^{2} \mathbf{p}}= & \sum_{q, g} \int_{0}^{1} d z D_{h / q, g}\left(z, \mu_{f r}\right) \\
& \times \frac{1}{z^{2}} \frac{d \sigma_{A B}^{q, g} \text { Quench }\left(\frac{\mathbf{p}}{z}\right)}{d y d^{2} \mathbf{p}}
\end{aligned}
$$

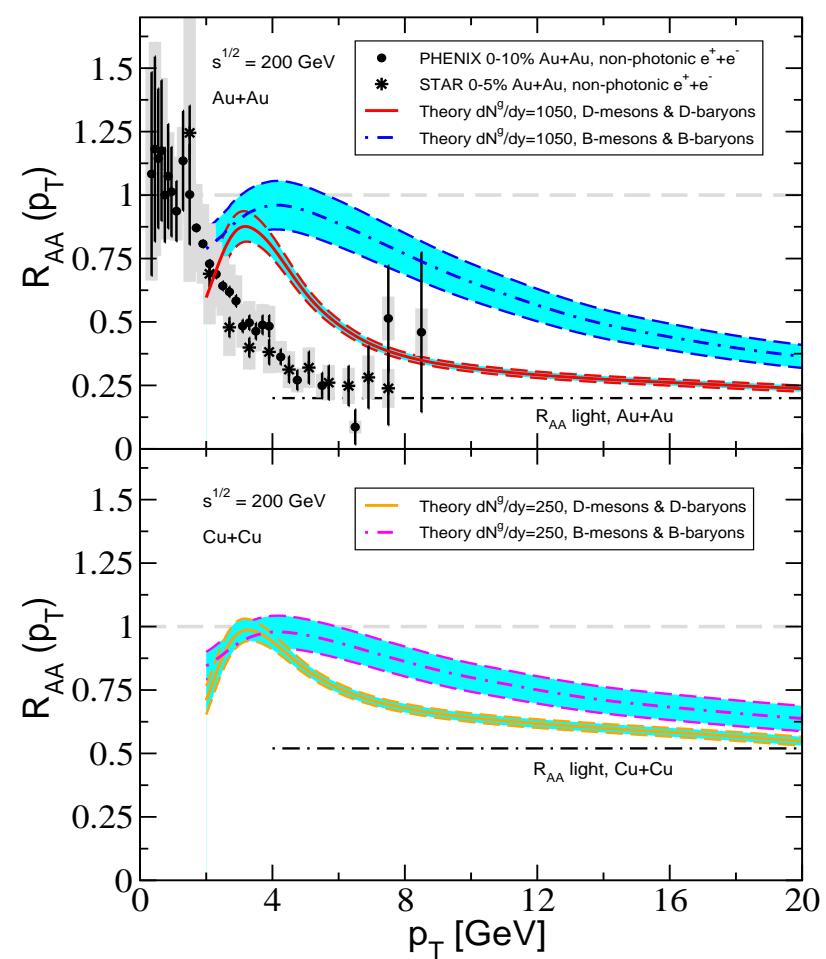

FIG. 2: Perturbative QCD calculation of the suppressed $D$ and $B$ meson production cross sections in central $\mathrm{Au}+\mathrm{Au}$ (top panel) and $\mathrm{Cu}+\mathrm{Cu}$ (bottom panel) collisions is compared to the magnitude of light pion quenching at $\sqrt{s_{N N}}=200 \mathrm{GeV}$. The effect of parton mass on the cold nuclear matter effects is illustrated via uncertainty bands. Non-photonic electron data from PHENIX [16] and STAR [17] is also shown.

where $z$ refers to the fraction of the light cone energy of the initial parton $q, g$, carried by the hadron $h$.

Eqs. (21), (12) and (13) summarize the traditional approach to jet quenching that has been very successful in the description of the observed attenuation of light hadron production in $\mathrm{A}+\mathrm{A}$ reactions. Figure@illustrates the level of agreement that can be achieved between the perturbative QCD theory, described in this section, and the experimental measurements of $\pi^{0}$ 33, 34] and $\frac{1}{2}\left(\pi^{+}+\pi^{-}\right)$[35] at RHIC. In central $\mathrm{Au}+\mathrm{Au}$ and $\mathrm{Cu}+\mathrm{Cu}$ reactions we used gluon rapidity densities $d N^{g} / d y=$ 1050 and $d N^{g} / d y=250$. Identical calculation for open heavy flavor production in heavy ion collisions at $\sqrt{s_{N N}}=200 \mathrm{GeV}$ is presented in Fig. 22 It is easy to see that Cronin enhancement plays an important role at intermediate $p_{T} \sim 4 \mathrm{GeV}$. The bands show the sensitivity of cold nuclear matter effects when we vary all parton masses in the range from 0 to $m_{c}, m_{b}$, respectively. At mid-rapidity, $y=0$, the effect of high-twist shadowing is small and the uncertainty band is dominated by initial state energy loss. Cold nuclear matter effects amplify the disparity between the heavy and light parton quenching. We have schematically illustrated the latter by showing the magnitude of pion attenuation from Fig. 1. 
In contrast, the experimental results on non-photonic electrons [16, 17] $R_{A A}^{e^{ \pm}} \sim R_{A A}^{\pi^{0}}, p_{T}>5 \mathrm{GeV}$ are in clear contradiction with the small quenching of $B$ mesons that give an increasingly important $\geq 50 \%$ contribution to non-photonic $e^{+}+e^{-}$in this region [7, 36]. An insight into the possible cause for this well-known by now discrepancy can be gained by examining Eq. (8). The small pion mass ensures that the parent light quarks and gluons fragment outside of the QGP in accord with the traditional picture of jet quenching. The large $D$ and $B$ meson mass, however, implies that charm and beauty quarks will fragment inside the hot and dense medium: $\tau_{\text {form }} \propto 1 / m_{h}^{2}$. Consequently, the competition between heavy meson dissociation and the $c$ and $b$ quark decay in the QGP is a likely physics mechanism that may naturally lead to attenuation of the beauty cross section as large as that for charm [7].

\section{DISTRIBUTION AND FRAGMENTATION FUNCTIONS OF HEAVY QUARKS}

Simulations of heavy quark fragmentation and dissociation in the QGP require knowledge of the corresponding parton decay and distribution probabilities. These are related to the wavefunctions of their parent or decay hadrons that can be represented as follows:

$$
\begin{aligned}
& \left|\vec{P}^{+} ; J\right\rangle=a_{h}^{\dagger}\left(\vec{P}^{+} ; J\right)|0\rangle=\sum_{n=2(3)}^{\infty} \int \prod_{i=1}^{n} \frac{d^{2} \mathbf{k}_{i}}{\sqrt{(2 \pi)^{3}}} \frac{d x_{i}}{\sqrt{2 x_{i}}} \\
& \times \psi\left(x_{i}, \mathbf{k}_{i} ; \alpha_{i}\right) \delta\left(\sum_{j=1}^{n} x_{j}-1\right) \delta^{2}\left(\sum_{j=1}^{n} \mathbf{k}_{j}\right) \\
& \times \mid \cdots a_{q_{i}}^{\dagger}\left(x_{q_{i}} \vec{P}^{+}+\mathbf{k}_{q_{i}}, \alpha_{q_{i}}\right) \cdots b_{\bar{q}_{j}}^{\dagger}\left(x_{\bar{q}_{j}} \vec{P}^{+}+\mathbf{k}_{\bar{q}_{j}}, \alpha_{\bar{q}_{j}}\right) \cdots \\
& \left.\cdots c_{g_{k}}^{\dagger}\left(x_{g_{k}} \vec{P}^{+}+\mathbf{k}_{g_{k}}, \alpha_{g_{k}}\right) \cdots\right\rangle .
\end{aligned}
$$

Here, $\overrightarrow{P^{+}} \equiv\left(P^{+}, \mathbf{P}\right)$ are the large light-cone momentum and transverse momentum components of the hadron, and the momenta of the partons are given by $\left(x_{i} P^{+}, x_{i} \mathbf{P}+\mathbf{k}_{i}\right)$. Our conventions for the quantization of the fermion and vector fields, including notation and commutation relations for the creation and annihilation operators $a^{\dagger}, a, \cdots$, are given in Appendix A In Eq. (14) $\alpha_{q_{i}}$ is a set of additional relevant quantum numbers, such as helicity and color. From the normalization of the meson state of fixed projection $\lambda$ of the total angular momentum $J$ :

$$
\left\langle\vec{P}^{+} ; J \mid \vec{P}^{+\prime} ; J\right\rangle=2 P^{+}(2 \pi)^{3} \delta\left(P^{+}-P^{+\prime}\right) \delta^{2}\left(\mathbf{P}-\mathbf{P}^{\prime}\right) \delta_{\lambda \lambda^{\prime}},
$$

an integral constraint on the norm of the light-cone wavefunctions $\psi\left(x_{i}, \mathbf{k}_{i} ; \alpha_{i}\right)$ can be derived.

In Eq. (14) proper construction of the non-perturbative lowest order Fock component in the expansion of a hadronic state in a quark and gluon basis, $q \bar{q}$ for mesons and $q q q$ for baryons, appears to be the most impor- tant. When properly constructed, higher order components that are generated via parton splitting, will have the correct quantum numbers through preservation of all relevant global symmetries in QCD. These components become dominant in the small momentum fraction $x$ region of sea quarks and gluons. On the other hand, for $x \sim \mathcal{O}(1)$ the momentum distribution of valence quarks can be determined from the relativistic quark model. For heavy quarks $(Q)$, perturbative splitting is suppressed by factors $\mathcal{O}\left(\Lambda_{Q C D}^{2} / M_{Q}^{2}\right)$ relative to light quarks $(q)$ and gluons $(g)$ and if we focus on the charm and beauty distribution and fragmentation functions the lowest order $Q \bar{q}$ (or $\bar{Q} q$ ) Fock component for heavy mesons will be a good starting point for our calculation:

$$
\begin{aligned}
& \left|\vec{P}^{+} ; J\right\rangle=\int \frac{d^{2} \mathbf{k}}{(2 \pi)^{3}} \frac{d x}{2 \sqrt{x(1-x)}} \frac{M(j)_{s_{1} s_{2}}}{\sqrt{2}} \frac{\delta_{c_{1} c_{2}}}{\sqrt{3}} \psi(x, \mathbf{k}) \\
& \times a_{Q}^{\dagger s_{1} c_{1}}\left(x \vec{P}^{+}+\mathbf{k}\right) b_{q}^{\dagger s_{2} c_{2}}\left((1-x) \vec{P}^{+}-\mathbf{k}\right)|0\rangle .
\end{aligned}
$$

In Eq. (16) $s_{1}, s_{2}$ refer to the spins of $Q$ and $\bar{q}$ and we have used the notation:

$$
\begin{aligned}
& M(P)_{s_{1} s_{2}}=(\chi)_{s_{1} s_{2}}=\left(\begin{array}{cc}
0 & 1 \\
-1 & 0
\end{array}\right)_{s_{1} s_{2}}, \\
& M(V)_{s_{1} s_{2}}=\sum_{i}\left(\epsilon_{i}(\lambda) \sigma_{i} \chi\right)_{s_{1} s_{2}},
\end{aligned}
$$

to ensure the correct spin structure of pseudoscalar ${ }^{1} S_{0}$ and vector ${ }^{3} S_{1}$ states and proper normalization [37]. A set of polarization vectors for $J=1$ in Eq. (18) in the meson rest frame is given by:

$$
\vec{\epsilon}_{ \pm 1}=\mp \frac{1}{\sqrt{2}}\left(\begin{array}{c}
1 \\
\pm i \\
0
\end{array}\right), \vec{\epsilon}_{3}=\left(\begin{array}{l}
0 \\
0 \\
1
\end{array}\right) \text {. }
$$

The color singlet structure is ensured by $\delta_{c_{1} c_{2}}$ where $c_{1}, c_{2}$ are the color indices of $Q$ and $\bar{q}$, respectively. Following Eq. (15) we can derive the normalization for each helicity $\lambda$ :

$$
\frac{1}{2(2 \pi)^{3}} \int d x d^{2} \mathbf{k}|\psi(x, \mathbf{k})|^{2}=1 .
$$

Note that the meson wavefunction can also be written in an instant form. For example, in the rest frame of the meson:

$$
\begin{aligned}
|\vec{P}=0 ; J\rangle_{h} & =\int \frac{d^{3} \vec{q}}{(2 \pi)^{3}} \frac{M(j)_{s_{1} s_{2}}}{\sqrt{2}} \frac{\delta_{c_{1} c_{2}}}{\sqrt{3}} f(\vec{q}) \\
& \times \sqrt{\frac{E_{h}}{2 E_{1} E_{2}}} a_{Q}^{\dagger s_{1} c_{1}}(\vec{q}) b_{q}^{\dagger s_{2} c_{2}}(-\vec{q})|0\rangle,
\end{aligned}
$$

where $\vec{q}$ represents the relative momentum between quarks and $f(\vec{q})$ is the Fourier transform of the meson wavefunction in position space. $E_{h}=\sqrt{\vec{P}^{2}+m_{h}^{2}}$ is the meson energy and $E_{i}=\sqrt{\vec{q}_{i}^{2}+m_{i}^{2}}$ are the quark energies. Conversion between the instant and light-cone 
forms is facilitated if harmonic oscillator, or Gaussian, approximation is made for the shape of the wavefunction $f(\vec{q})$ 38], which allows to easily ensure identical transverse width $\left\langle\mathbf{k}^{2}\right\rangle$ 7] for both forms.

In the light-cone gauge $A^{+}=0$, the heavy quark distribution function is given by [39]:

$$
\begin{aligned}
\phi_{Q / h}(x)= & \int \frac{d y^{-}}{2 \pi} e^{-i x P^{+} y^{-}} \\
& \times\left\langle P^{+}\left|\bar{\psi}_{Q}\left(y^{-}, \mathbf{0}\right) \frac{\gamma^{+}}{2} \psi_{Q}(0, \mathbf{0})\right| P^{+}\right\rangle,
\end{aligned}
$$

where $\psi_{Q}$ is the heavy quark field operator, $\left|P^{+}\right\rangle$are appropriately normalized hadronic states with large lightcone momentum $P^{+}$, and the average over the hadron spin/polarization states is implicit. For both the pseudoscalar and the vector cases, substituting Eq. (16) in the perturbative QCD definition, we proceed to a straightforward evaluation at tree level. We first carry out the contractions of the heavy and light quark creation and annihilation operators, perform the color sums and the integration over the internal kinematic variables to arrive at an intermediate result:

$$
\begin{aligned}
\phi_{Q / h}(x) \propto & \int \frac{d^{2} \mathbf{k}}{(2 \pi)^{3}} \frac{d x_{Q}}{2 x_{Q}} \int \frac{d y^{-}}{2 \pi} e^{-i\left(x-x_{Q}\right) P^{+} y^{-}} \\
& \times\left|\psi\left(x_{Q}, \mathbf{k}\right)\right|^{2} \frac{1}{2 J+1} \sum_{\lambda}\left(M(\lambda) M^{\dagger}(\lambda)\right)_{s_{1} s_{2}} \\
& \times \frac{1}{2} \bar{u}^{s_{1}}\left(x_{Q} P^{+}\right) \frac{\gamma^{+}}{2} u^{s_{2}}\left(x_{Q} P^{+}\right),
\end{aligned}
$$

where $u^{s}(p)$ are Dirac spinors defined in Appendix $\mathrm{A}$. With the polarization average yielding $\delta_{s_{1} s_{2}}$ and the spin sum resulting in $x_{Q} P^{+}$we readily obtain the final result:

$$
\phi_{Q / h}(x)=\frac{1}{2(2 \pi)^{3}} \int d x_{Q} d^{2} \mathbf{k}\left|\psi\left(x_{Q}, \mathbf{k}\right)\right|^{2} \delta\left(x_{Q}-x\right) .
$$

In contrast, the tree-level calculation of fragmentation of the heavy quark into hadron $h$, defined as [39]:

$$
\begin{aligned}
D_{h / Q}(z) & =z \int \frac{d y^{-}}{2 \pi} e^{i \frac{p^{+}}{z} y^{-}} \frac{1}{3} \operatorname{Tr}_{\text {color }} \frac{1}{2} \operatorname{Tr}_{\text {Dirac }} \frac{\gamma^{+}}{2} \\
& \times\left\langle 0\left|\psi\left(y^{-}, \mathbf{0}\right) a_{h}^{\dagger}\left(P^{+}\right) a_{h}\left(P^{+}\right) \bar{\psi}(0, \mathbf{0})\right| 0\right\rangle,
\end{aligned}
$$

can be shown to be proportional to $\left\langle 0\left|b_{q}^{\dagger}(\cdots) b_{q}(\cdots)\right| 0\right\rangle=$ 0 and, hence, vanishes. This result is also reinforced by the mismatch at tree-level between the large lightcone momentum of the parent heavy quark and the corresponding momentum of the heavy quark inside the meson. Since both $z=p_{h}^{+} / p_{Q}^{+} \leq 1$ and $x_{Q} \leq 1$, in the region of interest $D_{h / Q}(0<z<1) \equiv 0$.

The first non-trivial contribution to $D_{h / Q}(z)$ comes from the following matrix element that replaces the treelevel expression in Eq. (24):

$$
\begin{aligned}
& \langle 0| \psi_{Q}\left(y^{-}, \mathbf{0}\right) \int d^{4} y_{1}\left(-i g A_{\mu}^{\alpha} \bar{\psi}_{Q} \gamma^{\mu} T^{\alpha} \psi_{Q}\right) \int d^{4} y_{2}\left(-i g A_{\nu}^{\alpha} \bar{\psi}_{q} \gamma^{\nu} T^{\alpha} \psi_{q}\right) \times a_{Q}^{\dagger}\left(p_{1}\right) b_{q}^{\dagger}\left(p_{1}^{\prime}\right) b_{q}\left(p_{2}^{\prime}\right) a_{Q}\left(p_{2}\right) \\
& \times \int d^{4} y_{3}\left(-i g A_{\sigma}^{\beta} \bar{\psi}_{q} \gamma^{\sigma} T^{\beta} \psi_{q}\right) \int d^{4} y_{4}\left(-i g A_{\lambda}^{\beta} \bar{\psi}_{Q} \gamma^{\lambda} T^{\beta} \psi_{Q}\right) \bar{\psi}(0, \mathbf{0})|0\rangle
\end{aligned}
$$

and corresponds to the process with Feynman diagram shown in Fig. 3. $T^{\alpha}$ are the standard Gell-Mann matrices. Our approach is similar to the one outlined in [37, 40, 41] but in the calculation we retain $\vec{q}$, the $Q \bar{q}$ relative momentum distribution, in the matrix element. This will allow us to calculate the lowest order correction in $\vec{q}$ to $D_{h / Q}(z)$.

After converting the integral over the transverse momentum of the outgoing free light parton $p_{q}$ into an integral over the virtuality of the heavy quark we obtain the

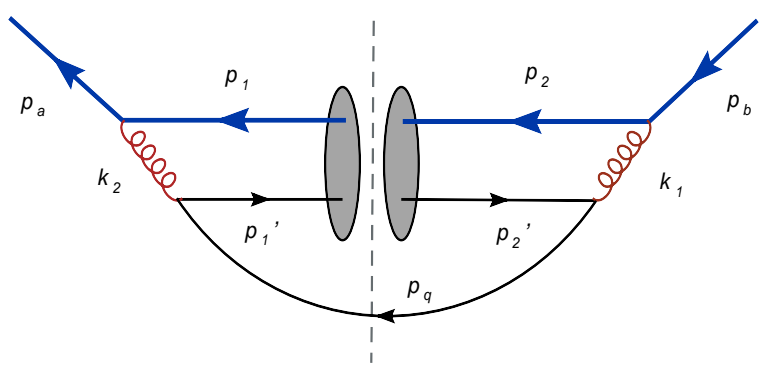

FIG. 3: Perturbative QCD calculation of heavy quark fragmentation matched to the lowest lying Fock state in the heavy meson wavefunction. 
heavy quark fragmentation functions:

$$
\begin{aligned}
& D_{h / Q}(z)=\int \frac{d x_{1} d^{2} \mathbf{k}_{1} \psi\left(x_{1}, \mathbf{k}_{1}\right)}{(2 \pi)^{3} 2 \sqrt{x_{1}\left(1-x_{1}\right)}} \frac{d x_{2} d^{2} \mathbf{k}_{2} \psi^{*}\left(x_{2}, \mathbf{k}_{2}\right)}{(2 \pi)^{3} 2 \sqrt{x_{2}\left(1-x_{2}\right)}} \\
& \frac{M(j)_{s_{1} s_{1}^{\prime}}}{\sqrt{2}} \frac{M(j)_{s_{2} s_{2}^{\prime}}}{\sqrt{2}} \int d s \theta\left(s-\frac{m_{h}^{2}}{z}-\frac{m_{q}^{2}}{1-z}\right) \frac{\alpha_{s}^{2} C_{F}^{2}}{3} \\
& \operatorname{Tr}\left[\gamma^{+} \frac{i}{\gamma \cdot p_{a}-m_{Q}} \gamma^{\mu} u_{s_{1}}\left(p_{1}\right) \bar{v}_{s_{1}^{\prime}}\left(p_{1}^{\prime}\right) \gamma^{\nu}\left(\gamma \cdot p_{q}+m_{q}\right) \gamma^{\sigma}\right. \\
& \left.v_{s_{2}^{\prime}}\left(p_{2}^{\prime}\right) \bar{u}_{s_{2}}\left(p_{2}\right) \gamma^{\lambda} \frac{i}{\gamma \cdot p_{b}-m_{q}} \Pi_{\mu \nu}\left(p_{a}-p_{q}\right) \Pi_{\sigma \lambda}\left(p_{b}-p_{q}\right)\right] \\
& \frac{1}{\operatorname{Tr}\left[\gamma^{+}(\gamma \cdot p)\right]} .
\end{aligned}
$$

Here, $\Pi_{\mu \nu}\left(p_{a}-p_{q}\right)=i\left(-g_{\mu \nu}+\left(n_{\mu} k_{\nu}+n_{\nu} k_{\mu}\right) /(n \cdot k)\right)$ is the gluon propagator, with $n^{\mu}=[0,1, \mathbf{0}]$ in light cone coordinates, and $s=p_{a}^{2}=p_{b}^{2}$, with $p_{a}=p_{b}=p_{Q}$. If we define:

$$
\begin{aligned}
& T(z)=M(j)_{s_{1} s_{1}^{\prime}} M(j)_{s_{2} s_{2}^{\prime}} \int d s \theta\left(s-\frac{m_{h}^{2}}{z}-\frac{m_{q}^{2}}{1-z}\right) \\
& \operatorname{Tr}\left[\gamma^{+} \frac{i}{\gamma \cdot p_{a}-m_{Q}} \gamma^{\mu} u_{s_{1}}(p 1) \bar{v}_{s_{1}^{\prime}}\left(p_{1}^{\prime}\right) \gamma^{\nu}\left(\gamma \cdot p_{q}+m_{q}\right) \gamma^{\sigma}\right. \\
& \left.v_{s_{2}^{\prime}}\left(p_{2}^{\prime}\right) \bar{u}_{s_{2}}\left(p_{2}\right) \gamma^{\lambda} \frac{i}{\gamma \cdot p_{b}-m_{q}} \Pi_{\mu \nu} \Pi_{\sigma \lambda}\right] \frac{1}{\operatorname{Tr}\left[\gamma^{+}(\gamma \cdot p)\right]},
\end{aligned}
$$

the heavy quark fragmentation functions into heavy mesons are then given by:

$$
D_{h / Q}(z)=\int \frac{d^{3} q_{1} f\left(\vec{q}_{1}\right)}{(2 \pi)^{3}} \frac{d^{3} q_{2} f^{*}\left(\vec{q}_{2}\right)}{(2 \pi)^{3}} T(z) .
$$

Note that the connection between the instant-form wavefunction $f\left(\vec{q}_{i}\right)$ and the light-cone wavefunction $\psi\left(x_{1}, \mathbf{k}_{1}\right)$ is discussed in detail in Appendix B. Thus, Eq. (28) is equivalent to Eq. (26).

The spin sums for the $Q \bar{q}{ }^{1} S_{0}(\mathrm{P})$ and ${ }^{3} S_{1}(\mathrm{~V})$ states can be derived, yielding:

$$
\begin{aligned}
& \sum_{s_{1}, s_{1}^{\prime}} u_{s_{1}}\left(p_{1}\right) \bar{v}_{s_{1}^{\prime}}\left(p_{1}^{\prime}\right) M(P)_{s_{1} s_{1}^{\prime}} \\
= & -\frac{\left(\not p_{1}+m_{Q}\right)\left(1+\gamma^{0}\right)\left(\not p_{1}^{\prime}+m_{q}\right) \gamma^{5}}{\sqrt{2\left(p_{1}^{0}+m_{Q}\right)} \sqrt{2\left(p_{1}^{\prime 0}+m_{q}\right)}}, \\
& \sum_{s_{1}, s_{1}^{\prime}} u_{s_{1}}\left(p_{1}\right) \bar{v}_{s_{1}^{\prime}}\left(p_{1}^{\prime}\right) M(V)_{s_{1} s_{1}^{\prime}} \\
= & \frac{\left(\not p_{1}+m_{Q}\right)\left(1+\gamma^{0}\right)\left(\not p_{1}^{\prime}+m_{q}\right) \notin_{p}(\lambda)}{\sqrt{2\left(p_{1}^{0}+m_{Q}\right)} \sqrt{2\left(p_{1}^{\prime 0}+m_{q}\right)}},
\end{aligned}
$$

where $\epsilon_{p}(\lambda)$ is the 4 -component polarization vector for a specific helicity projection $\lambda$ of the ${ }^{3} S_{1}$ state. In deducing the above expressions, we can first compute the spin sums in the rest frame of the heavy meson, then generalize them to an arbitrary frame.

Let us first neglect the effects of quark motion on the meson mass and assume in the rest frame that $M=$
$m_{Q}+m_{q}, r=m_{q} / M, 1-r=m_{Q} / M$. In a Lorentz boosted frame the light and heavy quark momenta can be written as:

$$
p_{1}=(1-r) p+q_{1 P}, p_{1}^{\prime}=r p-q_{1 P}
$$

In Eq. (31) $q_{i P}$ is the boosted relative momentum between the quarks in the lowest Fock state ( $\vec{q}$ at rest). A classic approximation [37, 40, 41] is to assume that the wavefunction $f(\vec{q})$ of the heavy meson as a bound state of two quarks goes rapidly to zero when $\vec{q} \neq 0$. The dominant contribution is, hence, given by the region $\vec{q} \sim 0$ and in this limit the spin sums in Eq. (29) and Eq. (30) can be reduced to:

$$
\begin{aligned}
& \sum_{s_{1}, s_{1}^{\prime}} u_{s_{1}}\left(p_{1}\right) \bar{v}_{s_{1}^{\prime}}\left(p_{1}^{\prime}\right) M(P)_{s_{1} s_{1}^{\prime}}=\frac{\sqrt{m_{Q} m_{q}}}{M} \gamma^{5}(\not p-M), \\
& \sum_{s_{1}, s_{1}^{\prime}} u_{s_{1}}\left(p_{1}\right) \bar{v}_{s_{1}^{\prime}}\left(p_{1}^{\prime}\right) M(V)_{s_{1} s_{1}^{\prime}}=-\frac{\sqrt{m_{Q} m_{q}}}{M}(\not p+M) \notin_{p}(\lambda),
\end{aligned}
$$

which are the same as the ones used in Refs. [37, 40, 41].

In this paper we employ the complete expressions for the spin sums, Eqs. (29) and (30), and calculate $T(z)$ defined in Eq. (27). This evaluation is technically very complex, with $\mathcal{O}\left(10^{4}\right)$ terms, and requires the use of packages for Dirac algebra manipulation [42]. Having obtained the full result, we can take the $q \rightarrow 0$ limit to obtain the lowest $\mathcal{O}\left(q^{0}\right)$ contribution to heavy quark fragmentation into the ${ }^{1} S_{0}$ state:

$$
\begin{aligned}
& T_{P}^{(0)}(z)= \\
& \frac{(z-1)^{2} z}{3 M^{3} r^{2}((r-1) z+1)^{6}}\left\{3(r-1)^{2}(2(r-1) r+1) z^{4}\right. \\
& +2(r-1)(r(18 r-19)+6) z^{3}+\left(68 r^{2}-74 r+21\right) z^{2} \\
& +(36 r-18) z+6\}
\end{aligned}
$$

and the one into the ${ }^{3} S_{1}$ state:

$$
\begin{aligned}
& T_{V}^{(0)}(z)=\frac{(z-1)^{2} z}{M^{3} r^{2}((r-1) z+1)^{6}} \\
& \left\{z \left((r-1)^{2}(2(r-1) r+3) z^{3}+2(r-1)(r(2 r-1)+4) z^{2}\right.\right. \\
& +6 r(2 r-1) z+9 z+4 r-6)+2\}
\end{aligned}
$$

respectively, which recover the results given by [37, 41].

Next, we proceed to evaluate the $\mathcal{O}\left(q^{1}\right)$ correction to the fragmentation function. To do so, we not only select the terms with 1-st power of $q_{1 P}$ and $q_{2 P}$, but also take the large $p_{Q}^{+}$limit (keeping the leading power of $p_{Q}^{+}$). We are able for the first time to obtain a meson structuredependent correction to the heavy quark fragmentation 
functions. For the ${ }^{1} S_{0}$ state or result reads:

$$
\begin{aligned}
& T_{P}^{(1)}(z)= \\
& \frac{(z-1)^{2} z^{2}}{6 M^{3}(1-r) r^{3}((r-1) z+1)^{8}}\left[\frac{p_{Q}^{+}}{q_{1 P}^{+}} \frac{\mathbf{q}_{1 T}^{2}}{M^{2}}+\frac{p_{Q}^{+}}{q_{2 P}^{+}} \frac{\mathbf{q}_{2 T}^{2}}{M^{2}}\right] \\
& \left\{3(r-1)^{4} r z^{6}+(r-1)^{3}(2 r+5) z^{5}+3(r-1)^{3}(4 r-7) z^{4}\right. \\
& -(r-1)(r(r(8 r-63)+88)-36) z^{3}+[r((81-28 r) r-90) \\
& \left.+32] z^{2}+((25-8 r) r-15) z+3\right\},
\end{aligned}
$$

while for the ${ }^{3} S_{1}$ state we get:

$$
\begin{aligned}
& T_{V}^{(1)}(z)=\frac{(z-1)^{2} z^{2}}{6 M^{3}(1-r) r^{3}((r-1) z+1)^{8}} \\
& \left\{\left(3 z^{4}\left(z^{2}+z+2\right) r^{5}-z^{3}\left(12 z^{3}+19 z^{2}+6 z-16\right) r^{4}\right.\right. \\
& +z^{2}\left(18 z^{4}+50 z^{3}-68 z^{2}+51 z-36\right) r^{3}-z\left(12 z^{5}+66 z^{4}\right. \\
& \left.-176 z^{3}+203 z^{2}-121 z+16\right) r^{2}+(z-1)^{2}\left(3 z^{4}+49 z^{3}\right. \\
& \left.\left.-59 z^{2}+50 z-6\right) r-(z-1)^{3}\left(11 z^{2}-13 z+9\right)\right) \frac{p_{Q}^{+}}{q_{1 P}^{+}} \frac{\mathbf{q}_{1 T}^{2}}{M^{2}} \\
& +\left(3 r^{6} z^{6}+r^{5}\left(-6 z^{2}+13 z+14\right) z^{4}-r^{4}\left(12 z^{3}+27 z^{2}\right.\right. \\
& +29 z-32) z^{3}+r^{3}\left(48 z^{4}-16 z^{3}+20 z^{2}-9 z-28\right) z^{2} \\
& -r^{2}\left(57 z^{5}-80 z^{4}+26 z^{3}+57 z^{2}-76 z+16\right) z \\
& -(z-1)^{3}\left(6 z^{3}-z^{2}-4 z+6\right)+r(z-1)^{2}\left(30 z^{4}-9 z^{3}\right. \\
& \left.\left.\left.-10 z^{2}+32 z-6\right)\right) \frac{p_{Q}^{+}}{q_{2 P}^{+}} \frac{\mathbf{q}_{2 T}^{2}}{M^{2}}\right\}
\end{aligned}
$$

In Eq. (36) we observe that for the triplet state the coefficients of $q_{1 P}$ and $q_{2 P}$ are different, which results from the different combinations of $\gamma$ matrices. For example, a typical term in the intermediate results for the ${ }^{3} S_{1}$ state may look like

$$
\operatorname{Tr}\left[A \not q_{1 P}\left(1+\gamma^{0}\right)(\not p+M) B(\not p+M)\right],
$$

where A and B are polynomials of Dirac matrices. This is not always equal to:

$$
\operatorname{Tr}\left[A(\not p+M) B(\not p+M)\left(1+\gamma^{0}\right) \not_{2 P}\right],
$$

even after exchanging the momenta $(1 \leftrightarrow 2)$. On the other hand, $q_{i T}, q_{i P}^{+}$(or $\vec{q}$ in the rest frame) are themselves internal integration variables with equal weights $\psi\left(x_{i}, \mathbf{k}_{\mathbf{i}}\right)$ (or $f\left(\vec{q}_{i}\right)$ in instant form), see Eqs. (26) and (28). Hence, Eq. (36) can be written in a symmetric form if desired.

Finally, we note that if the relative momentum of the light and heavy quarks is not neglected then in a boosted frame the large momentum of the meson in Eq. (31) will be partitioned proportional to the parton transverse

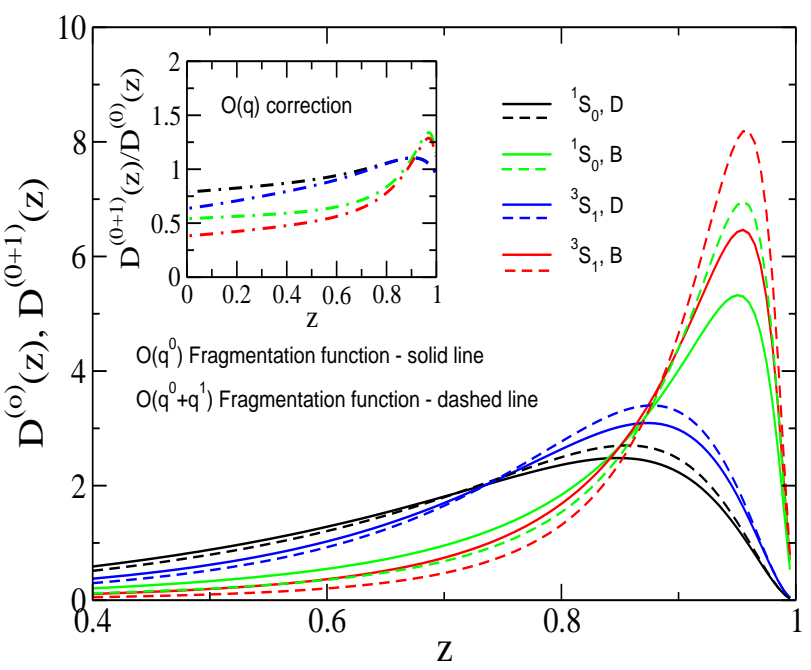

FIG. 4: Fragmentation functions of charm and beauty quarks into ${ }^{1} S_{0}$ and ${ }^{3} S_{1}$ heavy meson states. Solid lines are for negligible momentum spread between the parton constituents of $D$ and $B$. Dashed lines represent the first calculation for $\vec{q} \neq 0$. Insert shows the fractional correction that arises from the structure of the final-state hadrons for both the pseudoscalar and vector states.

mass, $m_{T Q}=\sqrt{m_{Q}^{2}+\left\langle\mathbf{k}^{2}\right\rangle}, m_{T q}=\sqrt{m_{q}^{2}+\left\langle\mathbf{k}^{2}\right\rangle}$. In this more general case $M=m_{T Q}+m_{T q}$ and $r=m_{T q} / M$, $1-r=m_{T Q} / M$. In this work we calculate $\left\langle\mathbf{k}^{2}\right\rangle=\left\langle\mathbf{q}^{2}\right\rangle$ from the light-cone wavefunctions of heavy mesons and use the corresponding values of $r$ both in the vacuum and at $T \neq 0$. This allows for a more consistent description of heavy quark fragmentation without treating $r$ as a phenomenological parameter.

With expressions for $T(z)$ at hand, after integrating over the relative momenta of $Q \bar{q}$ system in the rest frame of the heavy meson, we obtain the $z$-dependence of heavy quark fragmentation functions for the ${ }^{1} S_{0}$ and ${ }^{3} S_{1}$ states. Keeping in mind that the relative momentum is rather small and in the calculation $|q| \ll r M$ and $|q| \ll(1-r) M$ was implicit, we choose the upper bound of this integration to be $r M / 2$ due to the fact that $r<(1-r)$. The absolute normalization of $D_{h / Q}(z)$ has been fixed by the known branching ratios of the charm and beauty quarks into heavy mesons and baryons, such that $\sum_{i} \int_{0}^{1} D_{h_{i} / Q}(z) d z=1$.

Numerical results for $c$ and $b$ quark fragmentation into the ${ }^{1} S_{0}$ and the ${ }^{3} S_{1} D$ and $B$ mesons states, respectively, are shown in Fig. 4 The solid lines in the figure denote the standard $\mathcal{O}\left(q^{0}\right)$ calculation of $D_{h / Q}(z)$, in accord with [37, 41]. The dashed lines signify the addition of the $\mathcal{O}\left(q^{1}\right)$ correction, the first correction that arises from the internal structure of the final-state meson. The magnitude of the $\mathcal{O}\left(q^{1}\right)$ term is best seen in the insert of Fig. 4 in the ratio $D^{(0+1)}(z) / D^{0}(z)$. While on an absolute scale the correction to the shape of heavy quark fragmentation can be $\pm 50 \%$, it doesn't change apprecia- 
bly the position of the large- $z$ peak and, hence, will not alter the cross section for the observed $D$ and $B$ meson production. To facilitate the comparison between the FFs in the vacuum and at $T \neq 0$ we will only use the leading result for structureless hadrons, Eqs. (33) and (34).

\section{THE FATE OF HEAVY MESONS BELOW AND ABOVE $T_{c}$}

In this section we move beyond the vacuum $T=0$ case. Potential models have been quite useful in studying the non-perturbative aspects of QCD. In particular, for quarkonia one can put the potential model on firm grounds by using an effective field theory (NRQCD) [43]. More generally, heavy-light mesons like the $D$ and the $B$ and their excited states at zero temperature have been studied in detail 12, 13. by treating the light $\bar{q}$ as a Dirac particle moving in a confining potential set up by the heavy quark $Q$. In this section we investigate the boundstate wavefunctions for $D$ - and $B$-mesons at rest and in equilibrium with a thermal medium or in a co-moving plasma. These can be employed to calculate the PDFs and FFs for quarks/mesons, as discussed in Appendix B

Lattice QCD results for the free energy of a system at temperature $T$ containing two infinitely heavy quarks separated by a distance $r$ [15] have been used to extract the static potential between these quarks:

$V(r)=\left\{\begin{array}{cc}-\frac{\alpha}{r}+\sigma r, & r<r_{\text {med }}(T) \\ -\frac{\alpha_{1}(T) \exp (-\mu(T) r)}{r}+\sigma r_{\text {med }}(T), & r>r_{\text {med }}(T)\end{array}\right.$

Here, $r_{\text {med }}(T)$ signifies the temperature-dependent distance scale at which the medium breaks off the linear confining potential, $\alpha_{1}(T)$ represents the effective coupling at a given temperature, and $\mu(T)$ is the Debye screening mass. Interpolation is typically used to smooth out the sharpness in the potential at $r=r_{m e d}$ and we describe the interpolation below. This potential has been employed previously to study the fate of heavy-heavy bound states in the QGP formed at temperatures above $T_{c}$ 14, 44].

For future convenience, it is useful to separate the "vector" $\left(V_{v}(r)\right)$ and "scalar" $\left(V_{s}(r)\right)$ parts of the potential in Eq. (37). This is done as described for $T=0$ in [12, 13]. In Eq. (37) the term proportional to $\sigma$, which in the absence of a medium would be a confining linear potential, is taken to be scalar. The Coulomb part is naturally interpreted as the zeroth component of a vector potential. Thus we consider:

$$
\begin{aligned}
& V_{v}(r)=\left\{\begin{array}{cc}
-\frac{\alpha}{r} & r<r_{\text {med }} \\
-\frac{\alpha_{1} \exp (-\mu r)}{r} & r>r_{\text {med }}
\end{array},\right. \\
& V_{s}(r)=\left\{\begin{array}{cc}
\sigma r & r<r_{\text {med }} \\
\sigma r_{\text {med }} & r>r_{\text {med }}
\end{array} .\right.
\end{aligned}
$$

The typical parameters used in Eq. (37) are $\alpha=0.445, \quad \alpha_{1}(T)=\frac{1}{3 \pi} g^{2}(2 \pi T)$, and $\mu(T)=$
$1.417 \sqrt{1+\frac{N_{f}}{6}} g(2 \pi T) T$. For $T>1.1 T_{c}$, we use the perturbative two loop expression for $g(2 \pi T)$. For $T$ below $0.9 T_{c}$, where the perturbative expression for $g(2 \pi T)$ does not work, we take $g=2.05$ which corresponds to $\alpha=0.445$, and near $T_{c}$ we interpolate between the two. The strength of the confining potential is taken to be $\sigma=0.224 \mathrm{GeV}^{2}$ and $T_{c}=192 \mathrm{MeV}$. The value of $\sigma$ is $\approx 5 \%$ larger than in 44 and we take $M_{c}=1.34 \mathrm{GeV}$ and $M_{b}=4.79 \mathrm{GeV} \cdot r_{m e d}=\min \left(0.4 \frac{T_{c}}{T}, 1.1\right) \mathrm{fm}$, where $1.1 \mathrm{fm}$ is the typical string breaking scale [44]. $V_{s}(r)$ is smoothed out on a scale much smaller than $r_{m e d}(T)$. $V_{v}(r)$, following [44], is smoothed by interpolating between $\alpha / r$ at $r=$ $r_{m e d}(T)$ and $\alpha_{1} \exp (-\mu r) / r$ at $r=r_{1}(T)$, where $r_{1}(T)$ is larger than $r_{\text {med }}(T)$. For our calculations, we take $r_{1}(T)=2 r_{\text {med }}(T)$ for $T<1.1 T_{c}$ and $r_{1}(T)=1.25 \mathrm{fm}$ for $T>1.1 T_{c}$. This gives a slightly stronger potential than that used by 44 .

The presence of bound states of heavy-heavy systems, like charmonia and bottomonia, can be explored by solving the non-relativistic Schrödinger equation for the heavy quarks in the presence of the potential Eq. (37). Here, we only look at the $l=0$ wavefunction, for which the equation has a form,

$$
\frac{1}{2\left(m_{Q} / 2\right)} \frac{1}{r} \frac{\partial^{2} r \psi}{\partial r^{2}}+V(r) \psi=E \psi(r),
$$

where $m_{Q}$ is the mass of the heavy quark. We find that $J / \psi$ bound state solutions exist up to about $2 T_{c}$ though they have a binding energy, quite sensitive to the precise value of $\sigma$, much less than the temperature. The $\Upsilon$ disappears at much higher temperatures but its binding energy becomes smaller than $T$ near $1.5 T_{c}$. These values are larger than the values reported in [44] because of two reasons. First, the value of $\sigma$ is a little larger. Second, we use a slightly different interpolation to smooth the potential between $r<r_{\text {med }}$ and $r>r_{\text {med }}$. Table \shows the binding energy and sizes for the $J / \psi$ and the $\Upsilon$ at various temperatures above $T_{c}$.

To investigate the existence of heavy-light mesons in the QGP we need to solve the Dirac equation for a light

\begin{tabular}{c|c|c|c}
$T / T_{c}$ & $T(\mathrm{GeV})$ & $E_{b}(\mathrm{GeV})$ & $\langle r\rangle(\mathrm{fm})$ \\
\hline 1.2 & 0.230 & 0.042 & 0.722 \\
1.4 & 0.269 & 0.030 & 0.804 \\
1.6 & 0.307 & 0.024 & 0.869 \\
1.8 & 0.346 & 0.020 & 0.923 \\
2.0 & 0.384 & 0.017 & 0.968 \\
\hline \hline 1.2 & 0.230 & 0.344 & 0.222 \\
1.4 & 0.269 & 0.301 & 0.232 \\
1.6 & 0.307 & 0.273 & 0.241 \\
1.8 & 0.346 & 0.254 & 0.248 \\
2.0 & 0.384 & 0.241 & 0.254 \\
\hline
\end{tabular}

TABLE I: The binding energy and mean radius for $J / \psi$ (upper table) and $\Upsilon$ (lower table) as a function of the temperature. 
particle moving in the potential created by a heavy quark:

$$
H \psi=E \psi,
$$

where $\psi$ is the four-component Dirac spinor and $H$ is the Dirac Hamiltonian. $H$ has the following form:

$$
H=-i \gamma^{0} \gamma^{i} \partial_{i}+V_{v}(r)+\gamma^{0}\left(m+V_{s}(r)\right) .
$$

The eigenfunctions of the spherically symmetric Dirac Hamiltonian can be written as [12, 13, 45, 46]:

$$
\psi(\mathbf{r})=\frac{1}{r}\left(\begin{array}{c}
G(r) \\
i \sigma \cdot \hat{\mathbf{r}} F(r)
\end{array}\right) \mathcal{Y}_{j l s}^{j_{3}}
$$

where $\mathcal{Y}_{j l s}^{j_{3}}$ are angular functions that are obtained by the angular momentum addition of $s=\frac{1}{2}$ to $l$ giving $j$. The energy eigenequations for the radial wavefunctions $F(r)$ and $G(r)$ that we need to solve are as follows:

$$
\mid \begin{aligned}
& F^{\prime}(r)-\frac{\kappa}{r} F(r)=\left(-E+V_{v}(r)+m+V_{s}(r)\right) G(r) \\
& G^{\prime}(r)+\frac{\kappa}{r} G(r)=\left(E-V_{v}(r)+m+V_{s}(r)\right) F(r),
\end{aligned}
$$

where $\kappa= \pm(j+1 / 2)$. For the lowest energy states that we are interested in, $j=1 / 2$ and $\kappa=-1$. We solve Eq. (43) numerically using the "shooting method" [47]. Since the potential tends to a constant at large distances, we begin from an exponentially decaying solution at a very large distance. We solve the differential equation implied by the Dirac equation for this boundary condition and choose the value of the energy $E$ such that the value of the wavefunctions $F$ and $G$ at $r=0$ is 0 .

Since the heavy-light mesons are larger in size when compared to quarkonia, it is natural to expect that they will be affected more severely by color screening. Indeed, for a light current quark mass of about $0.005 \mathrm{GeV}$, we don't find even very weakly bound states for $T>T_{c}$. However, this conclusion is quite sensitive to the parameters chosen above. For example, by simply changing $r_{\text {med }}$ from $0.4 \frac{T}{T_{c}}$ fm to $0.45 \frac{T}{T_{c}}$ fm [14] we find bound states up to $\sim 1.2 T_{c}$, albeit with binding energies $E_{b}$ about 50 times smaller than $T$. A quark moving in the

\begin{tabular}{c|c|c|c}
$T\left(T_{c}\right)$ & $T(\mathrm{GeV})$ & $E_{b}(\mathrm{GeV})$ & $\sqrt{\left\langle r^{2}\right\rangle}(\mathrm{fm})$ \\
\hline 0 & 0 & 0.730 & 0.468 \\
$0.2 T_{c}$ & 0.038 & 0.733 & 0.466 \\
$0.4 T_{c}$ & 0.077 & 0.611 & 0.464 \\
$0.6 T_{c}$ & 0.115 & 0.256 & 0.501 \\
$0.8 T_{c}$ & 0.154 & 0.098 & 0.632 \\
$1.0 T_{c}$ & 0.211 & 0.043 & 0.785 \\
$1.2 T_{c}$ & 0.230 & 0.031 & 0.970 \\
$1.4 T_{c}$ & 0.269 & 0.017 & 1.263 \\
$1.6 T_{c}$ & 0.307 & 0.009 & 1.636 \\
\hline
\end{tabular}

TABLE II: Properties of the $D$ and $B$ meson bound-state solutions taking the effective light quark mass to be $m(T) / \sqrt{2}$. These persist up to temperatures $\approx 1.6 T_{c}$.

\begin{tabular}{c|c|c|c}
$T\left(T_{c}\right)$ & $T(\mathrm{GeV})$ & $E_{b}(\mathrm{GeV})$ & $\sqrt{\left\langle r^{2}\right\rangle}(\mathrm{fm})$ \\
\hline$\cdots$ & $\cdots$ & $\cdots$ & $\cdots$ \\
$1.0 T_{c}$ & 0.211 & 0.060 & 0.670 \\
$1.2 T_{c}$ & 0.230 & 0.047 & 0.797 \\
$1.4 T_{c}$ & 0.269 & 0.032 & 0.933 \\
$1.6 T_{c}$ & 0.307 & 0.024 & 1.044 \\
$1.8 T_{c}$ & 0.346 & 0.020 & 1.119 \\
$2.0 T_{c}$ & 0.384 & 0.018 & 1.158 \\
\hline
\end{tabular}

TABLE III: Properties of the bound $D$ and $B$ meson states taking the effective light quark mass to be $m(T)$.

QGP will have an effective thermal mass larger than its bare mass $m_{q}$. For $T>T_{c}$ perturbative calculations give $m(T)=\sqrt{\frac{C_{F}}{4}[g(2 \pi T) T]^{2}+m_{q}^{2}}$. For $T<T_{c}$ this form is not valid and we simply interpolate the perturbative value above $T_{c}$ to the bare mass at $T=0$. One would expect the effective mass of a quark moving under the influence of a potential in the thermal medium to lie somewhere between $m_{q}$ and $m(T)$. For example, results obtained by solving the Dirac equation for the light quark mass taken to be $m(T) / \sqrt{2}$ are given in Table II. We remark that the very small rise in the binding energy that can be seen in Table II as we increase the temperature from $T=0$ to $0.2 T_{c}$ is an artifact of the interpolation we have used for the thermal mass below $T_{c}$ and is not numerically significant to affect our results. The use of light quark mass equal to $m(T)$ gives boundstate solutions with weak binding energies up to even above $2 T_{c}$, see Table III. We also establish the existence of $D$ and $B$ meson bound-state solutions well above the phase transition temperature for constituent light quark masses and/or for the "maximum confining potential" compatible with lattice data: all the way up to $\sim 2 T_{c}$ for $m_{q}=m(T) / \sqrt{2}$ and $\sim 3 T_{c}$ for $m_{q}=m(T)$.

The bound-state wavefunctions, calculated at temperatures $T=0 T_{c}, 0.4 T_{c}, 0.8 T_{c}, 1.2 T_{c}$ with the light quark mass taken to be $m(T) / \sqrt{2}$ are employed to evaluate the PDFs and FFs of heavy quarks in a co-moving thermal medium from Eqs. (23) and (26) and our results are presented in Fig. The distribution functions $\phi_{Q / H}(x)$ become narrower in $x$ as we increase the temperature, which is intuitively expected because the wavefunctions become broader in position space. In contrast the peak position and width of the decay probabilities are determined by the boost parameter $r$. These remain nearly constant because the decrease in the mean transverse momentum squared is largely compensated by the growth in the thermal mass. Hence, we have used an arbitrary scale factor $s$ to better separate the curves in the bottom panel of Fig. 5. We remark that the double-peak structure arises from the decay of the excited ${ }^{3} S_{1}$ states into ${ }^{1} S_{0}$ states which effectively shifts the momentum fraction $z$ to lower values.

To summarize, as a rule we find that $D$ and $B$ meson bound-state solutions persist above $T_{c}$. Their small binding energy and large radius, however, will greatly 


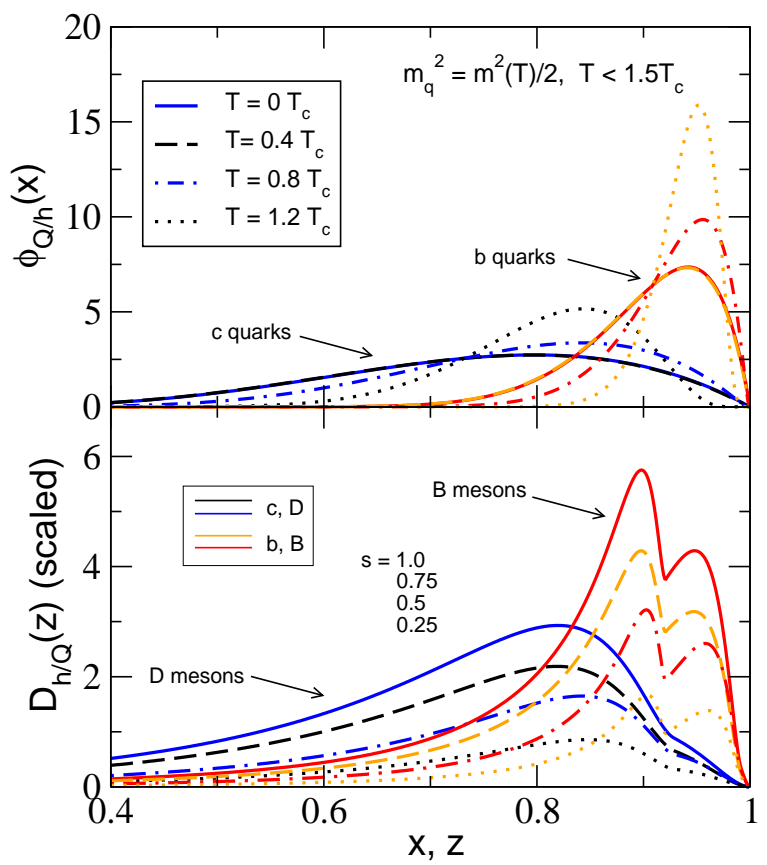

FIG. 5: The evolution of PDFs $\phi_{Q / H}(x)$ (top panel) and the FFs $D_{H / Q}(z)$ (bottom panel) with $T$. Blue/black lines refer to charm while red/orange lines refer to beauty. A scale factor $s$ has been used to better separate the decay probabilities.

facilitate their subsequent dissolution in the presence of interactions.

\section{APPLICATION TO HEAVY MESON PRODUCTION IN HEAVY ION COLLISIONS}

Heavy flavor dynamics in dense QCD matter critically depends on the time scales involved in the underlying reaction. Two of these timescales, $\tau_{0}$ and $L_{Q G P}$, can be related to the nuclear geometry, the QGP expansion, and the properties of bulk particle production 32]. They signify the onset and disappearance of QGP-induced effects and determine, in relation to the formation time Eq. (8), whether the suppression of the observed cross section arises predominantly at the hadronic $\left(\tau_{\text {form }} \ll L_{Q G P}\right)$ or partonic $\left(\tau_{\text {form }} \geq L_{Q G P}\right)$ level. As discussed in the previous section, the dissociation of $D$ and $B$ mesons in the vicinity of $T_{c}$ can be facilitated by their small binding energy, or, equivalently, their broad wavefunction in coordinate space. Whether such thermal effects take place in practice, however, depends on the time they need to develop. We can roughly estimate this time by boosting the expanded size of the hadron, $\approx 2 \sqrt{\left\langle r^{2}\right\rangle}$ from Table II by the $\gamma=1 / \sqrt{1-v^{2}}$ factor. When compared to $\tau_{\text {form }}$, which is determined by the virtuality in the parton decay process, this time is large and suggests that the fragmentation component of the heavy meson dynamics in heavy ion collisions may not be affected by the QGP. In what follows we will study this "instant wavefunction limit" in detail. Before we proceed we remark that in Appendix C we have also estimated the correction to the $\mathrm{FF} D_{h / Q}(z)$ from the pick-up of a light thermal quark strictly in the fragmentation region $0<z<1$ and found this effect to be small, $\leq 3 \%$ and $15 \%$ for $D$ and $B$ mesons at $p_{T} \geq 4 \mathrm{GeV}$, respectively.

A meson that is formed and propagates inside the medium will undergo collisional broadening and dissociation [7, 8]. The dissociation rate can be evaluated as follows:

$$
\frac{1}{\left\langle\tau_{\text {diss }}\left(p_{T}, t\right)\right\rangle}=\frac{\partial}{\partial t} \ln \left(1-P_{s}\left(p_{T}, m_{Q}, t\right)\right),
$$

where the survival probability is given by the overlap of the initial wavefunction $\psi_{0}$ with the wavefunction at time $t, \psi_{f}$ :

$$
P_{s}\left(\frac{\mu^{2} L}{\lambda_{q}} \xi\right)=\left|\int d^{2} \mathbf{k} d x \psi_{f}^{*}(\mathbf{k}, x) \psi_{0}(\mathbf{k}, x)\right|^{2},
$$

Details of the diagrammatic calculation and resummation of the interactions that lead to the final-state broadening of the $D$ and $B$ meson wavefunctions, $\psi_{0}(\mathbf{k}, x) \rightarrow$ $\psi_{f}(\mathbf{k}, x)$, can be found in [7]. We here remark that the harmonic oscillator wavefunction form, discussed in Appendix B. allows for an analytic evaluation of this broadening. As in Eq. (4), $\frac{\mu^{2} L}{\lambda_{q}} \xi$ is proportional to the cumulative transverse momentum transfer from the medium to the propagating system and is here calculated for Bjorken-expanding QGP. Formation rates are calculated by taking into account all final-state heavy meson states

$$
\begin{aligned}
\frac{1}{\left\langle\tau_{\text {form }}\left(p_{T}, t\right)\right\rangle}= & {\left[\sum_{i} \int_{0}^{1} d z D_{H_{i} / Q}(z)\right.} \\
& \left.\times \tau_{\text {form }}^{i}\left(z, p_{T}, m_{Q}, t\right)\right]^{-1} .
\end{aligned}
$$

Employing the above calculated rates, Eqs. (44) and (46), the concurrent processes of $c$ and $b$ quark fragmentation and $D$ and $B$ meson dissociation are described by the following set of rate equations [7]:

$$
\begin{aligned}
& \partial_{t} f^{Q}\left(p_{T}, t\right)=-\frac{1}{\left\langle\tau_{\text {form }}\left(p_{T}, t\right)\right\rangle} f^{Q}\left(p_{T}, t\right) \\
& +\frac{1}{\left\langle\tau_{\text {diss }}\left(p_{T} / \bar{x}, t\right)\right\rangle} \int_{0}^{1} d x \frac{1}{x^{2}} \phi_{Q / H}(x) f^{H}\left(p_{T} / x, t\right) \\
& \partial_{t} f^{H}\left(p_{T}, t\right)=-\frac{1}{\left\langle\tau_{\text {diss }}\left(p_{T}, t\right)\right\rangle} f^{H}\left(p_{T}, t\right) \\
& +\frac{1}{\left\langle\tau_{\text {form }}\left(p_{T} / \bar{z}, t\right)\right\rangle} \int_{0}^{1} d z \frac{1}{z^{2}} D_{H / Q}(z) f^{Q}\left(p_{T} / z, t\right) .
\end{aligned}
$$

Here, $f^{Q}\left(p_{T}, t\right)\left(f^{H}\left(p_{T}, t\right)\right)$ is the differential cross section to find a quark $Q$ (hadron $H$ ) with a fixed rapidity $y$ and transverse momentum $p_{T}$, at a time $t$. We have suppressed the rapidity dependence for clarity of notation. Note that the reason for which the asymptotic $t \rightarrow \infty$ 


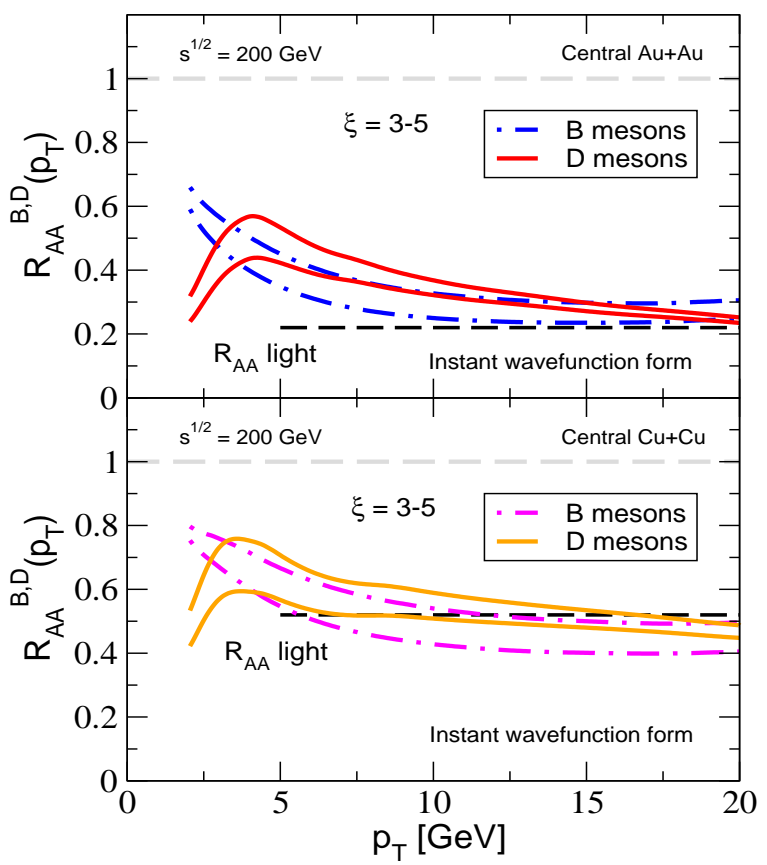

FIG. 6: Suppression of $D$ and $B$ hadron production from meson dissociation and heavy quark quenching in central $\mathrm{Au}+\mathrm{Au}$ (top panel) and $\mathrm{Cu}+\mathrm{Cu}$ collisions (bottom panel) at $\sqrt{s_{N N}}=200 \mathrm{GeV}$ at RHIC.

solution will exhibit suppression of the cross sections is that both fragmentation and dissociation processes emulate energy loss by shifting the quarks/hadrons to lower momenta. Under certain simplifying assumptions the system of equations, Eqs. (47) and (48), can be solved analytically, as shown in Appendix D.

At present, there is no reliable way of incorporating the fluctuations in partonic energy loss in rate or transport equations. Therefore, we include the early-time heavy quark inelastic scattering effects approximately as a quenched initial condition:

$$
\mid \begin{aligned}
& f^{Q}\left(p_{T}, t\right)=\frac{d \sigma^{Q}(t)}{d y d^{2} \mathbf{p}}, f^{Q}\left(p_{T}, 0\right)=\frac{d \sigma^{Q, \text { Quench }}}{d y d^{2} \mathbf{p}} \\
& f^{H}\left(p_{T}, t\right)=\frac{d \sigma^{H}(t)}{d y d^{2} \mathbf{p}}, f^{H}\left(p_{T}, 0\right)=0 .
\end{aligned}
$$

Here, the attenuated partonic spectrum $\frac{d \sigma^{Q}, \text { Quench }}{d y d^{2} \mathbf{p}}$ is calculated differentially versus $p_{T}$ using Eq. (12). The relevant mean quenching time - the time that the physical system of interest spends in a quark state - can be estimated from the analytic solution for $f^{Q}\left(p_{T}, t\right), f^{H}\left(p_{T}, t\right)$ given in Appendix D. On the other hand, $\tau_{\text {form }}$ represents the important stage until the first fragmentation of the heavy quark into heavy meson. We use the average of these two results to calculate the initial condition, Eq. (49).

We integrate numerically the above set of coupled ordinary differential equations and use the same initial soft gluon rapidity density $d N^{g} / d y$ as in the simulations of

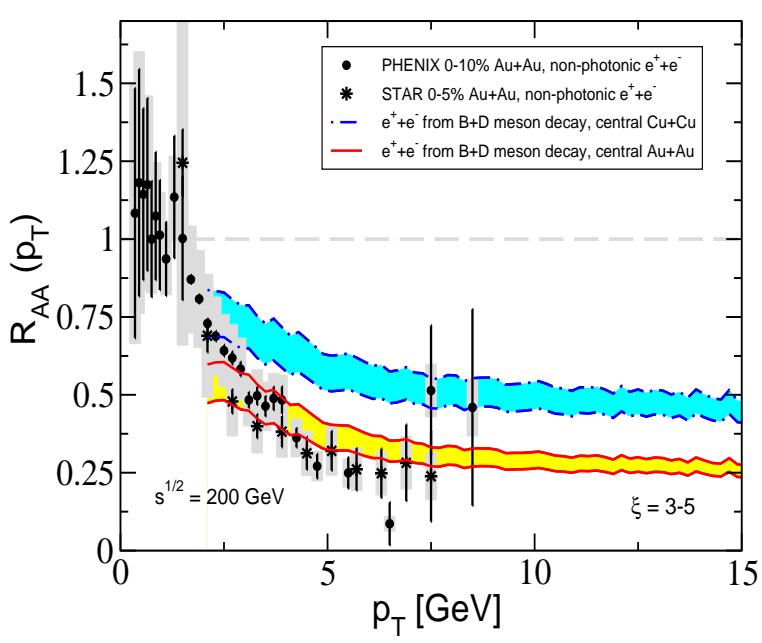

FIG. 7: Nuclear modification for the single non-photonic electrons in central $\mathrm{Au}+\mathrm{Au}$ and $\mathrm{Cu}+\mathrm{Cu}$ collisions at RHIC. Data is from PHENIX [16] and STAR [17] collaborations.

$\pi^{0}$ quenching. The corresponding suppression of open heavy flavor in $\sqrt{s_{N N}}=200 \mathrm{GeV}$ central $\mathrm{Au}+\mathrm{Au}$ and $\mathrm{Cu}+\mathrm{Cu}$ collisions at $\mathrm{RHIC}$ is shown in the top and bottom panels of Fig. 6, respectively. For $D$ mesons the Cronin effect is clearly visible around $p_{T} \sim 4 \mathrm{GeV}$ and this is a notable difference from our previous study 7] where initial-state $k_{T}$ diffusion was not included. For $B$ mesons the change in heavy quark velocity $\beta_{Q}$, which controls the $Q \bar{q}(\bar{Q} q)$ broadening $\propto \beta_{Q} \frac{\mu_{0}^{2}}{\lambda_{0}} \xi \ln \frac{\tau}{\tau_{0}}$ and dissociation, results in the characteristic rapid decrease in $R_{A A}^{B}\left(p_{T}\right)$ in this part of phase space. In both gold and copper reactions at RHIC the suppression $R_{A A}^{B} \approx R_{A A}^{D}$ for $p_{T}>4 \mathrm{GeV}$ and these approach the quenching of light hadrons for $p_{T}>10 \mathrm{GeV}$. It should be noted that while for the $D$ mesons we observe a transition from collisional dissociation to partonic energy loss in the studied kinematic domain, for B meson at RHIC the competing hadronic processes, Eqs. (47) and (48), still play the dominant role.

Our theoretical results, presented in Fig. 6, are most relevant to the future vertex detector upgrades at RHIC that will ensure direct and separate measurements of the $D$ and $B$ mesons. These new experimental data will then allow to pinpoint the mechanisms of heavy flavor suppression in the QGP. Indirect studies of $D$ and $B$ meson attenuation are currently carried out through the semileptonic decays of the charm and beauty hadrons. We use the PYTHIA event generator 48] to simulate the full kinematics of these Dalitz decays in $\mathrm{p}+\mathrm{p}$ and $\mathrm{A}+\mathrm{A}$ reactions. The nuclear modification ratio $R_{A A}^{e}\left(p_{T}\right)$ of inclusive non-photonic electrons is presented in Fig. 7 for $\sqrt{s_{N N}}=200 \mathrm{GeV}$ collisions at RHIC. Predictions for central $\mathrm{Cu}+\mathrm{Cu}$ collisions are also shown for comparison to upcoming STAR data. We remark that the Cronin effect included in our study changes the abso- 


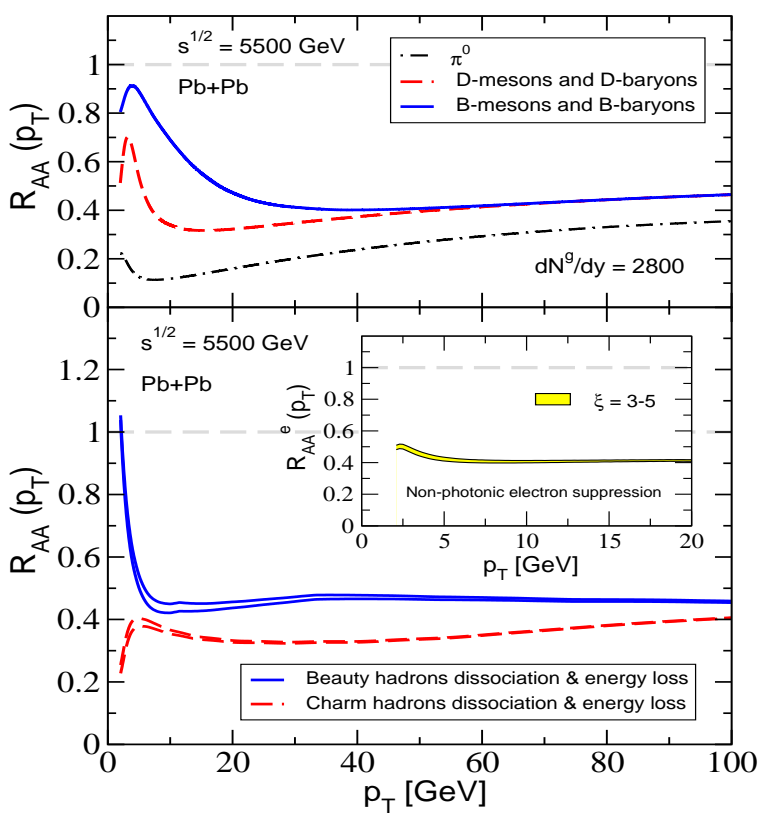

FIG. 8: Suppression of $D$ and $B$ meson production in central $\mathrm{Pb}+\mathrm{Pb}$ collisions at $\sqrt{s_{N N}}=5500 \mathrm{GeV}$ at the LHC in two different scenarios. The top panel shows the quenching of heavy hadrons only due to partonic energy loss. The bottom panel gives $R_{A A}$ for $D$ s and $B$ s with partonic energy loss as well as collisional dissociation of heavy meson. Insert shows the corresponding attenuation of non-photonic electrons in a limited $p_{T}$ range.

lute scale of the differential heavy quark cross section at intermediate $p_{T}$. Consequently, to obtain a good description of the non-photonic $e^{+}+e^{-}$quenching results from PHENIX [16] and STAR [17] we use $\xi=3-5$, approximately $50 \%$ larger than in our previous study 7] but still compatible with the enhancement of the parton broadening that comes from the power-law momentum transfer tails of the in-medium Moliere scattering.

Results for the suppression of open heavy flavor final states in central $\mathrm{Pb}+\mathrm{Pb}$ collisions at $\sqrt{s_{N N}}=5500 \mathrm{GeV}$ at the LHC in a medium of soft gluon rapidity density $d N^{g} / d y=2800$ are presented in Fig. 8 The top panel illustrates the effect of partonic energy loss with $R_{A A}^{B}\left(p_{T}\right)=R_{A A}^{D}\left(p_{T}\right)$ for $p_{T}$ about and above $50 \mathrm{GeV} . D$ and $B$ meson quenching approaches the suppression level of light hadrons only at very high transverse momentum due to the significant gluon fragmentation component to pion production in this kinematic region. The bottom panel shows the attenuation of open heavy flavor when we include collisional dissociation in the medium. The most important feature of our results, when compared to traditional jet quenching studies, is that at the LHC $R_{A A}^{B}\left(p_{T}\right) \approx R_{A A}^{D}\left(p_{T}\right)$ at a much lower $p_{T} \simeq 10 \mathrm{GeV}$. The nuclear modification factor of non-photonic electrons is also shown for completeness in the insert of Fig. 8. By comparing the top and bottom panels of Fig. 8 we con- clude that only experiments at the LHC will have the transverse momentum coverage to fully explore heavy flavor dynamics in the QGP. We finally remark that the small difference in the suppression of $D$ and $B$ mesons at $p_{T} \sim 50 \mathrm{GeV}$ is from the interplay of two quenching mechanisms - meson dissociation and parton energy loss. This difference disappears at $p_{T} \sim 150 \mathrm{GeV}$.

\section{CONCLUSIONS}

Detailed theoretical studies have shown that partonic energy loss of heavy quarks in the hot QGP [6] cannot explain the large suppression of non-photonic electrons observed by PHENIX [16] and STAR 17 collaborations in central $\mathrm{Au}+\mathrm{Au}$ collisions at $\sqrt{s_{N N}}=200 \mathrm{GeV}$ per nucleon pair. This discrepancy can naturally be resolved if collisional dissociation of heavy mesons that tend to form inside the QGP is taken into account 7]. In this paper we reported first results from an approach that attempts to combine charm and beauty quark quenching with $D$ and $B$ meson inelastic breakup processes with the goal of describing open heavy flavor production in nucleus-nucleus collisions over the full $p_{T}$ range that will be experimentally accessible at RHIC and the LHC. The treatment of cold nuclear matter effects for massive final state mesons was brought on par with that for pions and photons [28] and the Cronin enhancement at transverse momenta $\sim 4 \mathrm{GeV}$ was found to be the most important. To put studies of heavy meson formation and dissociation in the plasma [7, 8] on firmer theoretical ground we investigated in the framework of potential models 14 the existence of such bound states in the vicinity of $T_{c}$. We found that while the temperature at which $D$ and $B$ mesons cease to form depends on the details of the inmedium quark-antiquark potential and the light quark mass, as a rule bound states survive well above $T_{c}$. Using the light-cone description of hadrons [11] and the operator definitions of distribution and decay probabilities from factorized perturbative QCD [39] we calculated the charm and beauty PDFs and FFs in a co-moving plasma.

In the instant wavefunction approximation, relevant to an out-of-equilibrium jet propagation through the medium when the timescale for the onset of thermal effects exceeds $\tau_{\text {form }}$, we evaluated the $D$ and $B$ meson cross section suppression from partonic and hadronic interactions in the QGP. We found that for $p_{T}>4 \mathrm{GeV}$ at RHIC and $p_{T}>10 \mathrm{GeV}$ at the LHC $R_{A A}^{B}\left(p_{T}\right) \approx$ $R_{A A}^{D}\left(p_{T}\right)$ and the transverse momentum dependence of this attenuation is weak. In our study meson dissociation played a dominant role up to $p_{T} \sim 10 \mathrm{GeV}$ for open charm and up to $p_{T} \sim 30 \mathrm{GeV}$ for open beauty hadrons. To obtain a good description of the non-photonic electron suppression in $\mathrm{Au}+\mathrm{Au}$ collisions at RHIC after the inclusion of Cronin enhancement 22] approximately 50\% larger collisional broadening [23] that leads to the meson breakup was required. Predictions were given for the 
$D$ and $B$ hadron and the corresponding non-photonic electron quenching in $\mathrm{Cu}+\mathrm{Cu}$ and $\mathrm{Pb}+\mathrm{Pb}$ collisions at RHIC and the LHC, respectively, that will very soon be confronted by data. We conclude by emphasizing that while charm quark/meson dynamics in the medium is well within the reach of RHIC experiments, for a comprehensive and definitive study which can disentangle the medium effects on beauty quarks/mesons not only the enhanced cross sections but also the extended $p_{T}$ reach at LHC experiments will play a critical role.

\section{Acknowledgments}

This research is supported by the US Department of Energy, Office of Science, under Contract No. DE-AC5206NA25396 and in part by the LDRD program at LANL, the NNSF of China and the MOE of China under Project No. IRT0624.

\section{APPENDIX A: NOTATION AND QUANTIZATION OF QUARK AND GLUON FIELDS}

To quantize the theory on the light cone we choose the light-cone time as $x^{+}=(1 / \sqrt{2})\left(x^{0}+x^{3}\right)$. The light-cone "spatial" components are taken as $\left(x^{-}, \mathbf{x}\right)$ where $x^{-}=$ $(1 / \sqrt{2})\left(x^{0}-x^{3}\right)$ and $\mathbf{x}$ is a two component spatial vector with $(\mathbf{x})^{1}=x^{1}$ and $(\mathbf{x})^{2}=x^{2}$. With this definition, the dot product between two four vectors $a$ and $b$ has the form $a \cdot b=a^{+} b^{-}+a^{-} b^{+}-\mathbf{a} \cdot \mathbf{b}$. We use the notation where $\vec{a}$ stands for the spatial components of a four vector $a^{\mu}$, and the 3-tuple $\vec{a}^{+}=\left(a^{+}, \mathbf{a}\right)$.

For quarks of every flavor and color there is a corresponding Dirac field $\psi_{c f}(x)$. In what follows, we will suppress the color index $c$ and the flavor index $f$ for simplicity. The free field Lagrangian for $\psi$ is given by:

$$
\mathcal{L}=\bar{\psi}(i \not \partial-m) \psi,
$$

where $\not \partial=\gamma^{\mu} \partial_{\mu}$. $\gamma^{\mu}$ are Dirac matrices satisfying $\left\{\gamma^{\mu}, \gamma^{\nu}\right\}=2 \eta^{\mu \nu}$. We write the Dirac matrices in the chiral representation:

$$
\gamma^{0}=\left(\begin{array}{ll}
0 & 1 \\
1 & 0
\end{array}\right), \gamma^{i}=\left(\begin{array}{cc}
0 & \sigma^{i} \\
-\sigma^{i} & 0
\end{array}\right) \text { for } i=1,2,3 .
$$

The light-cone Dirac matrices are defined by the relations $\gamma^{ \pm}=(1 / \sqrt{2})\left(\gamma^{0} \pm \gamma^{3}\right)$, and $\gamma^{i}=\gamma^{i}$ for $i=1,2$.

The equation of motion for $\psi$ is simply

$$
(i \not \partial-m) \psi(x)=0 \text {. }
$$

We look for plane wave solutions of form $u(p) \exp (-i p \cdot x)$ and $v(p) \exp (i p \cdot x)$ with $p^{+}>0$. To satisfy the equation of motion the four momentum $p$ should be on shell. In addition, the Dirac spinors $u(p)$ and $v(p)$ satisfy the following equations:

$$
(\not p-m) u(p)=0, \quad(\not p+m) v(p)=0 .
$$

The solutions to these equations can be written a nice form, which we use in the calculation of the heavy quark fragmentation functions:

$$
\begin{aligned}
& u(p, s)=\frac{1}{\sqrt{2\left(p^{0}+m\right)}}\left(\begin{array}{c}
(p \cdot \sigma+m) \xi_{s}^{u} \\
(p \cdot \bar{\sigma}+m) \xi_{s}^{u}
\end{array}\right), \\
& v(p, s)=\frac{-\gamma^{5}}{\sqrt{2\left(p^{0}+m\right)}}\left(\begin{array}{c}
(p \cdot \sigma+m) \xi_{s}^{v} \\
(p \cdot \bar{\sigma}+m) \xi_{s}^{v}
\end{array}\right),
\end{aligned}
$$

where $\xi_{s}$ is a two dimensional unit vector, which is different for $u$ and $v$ and depends on the chosen representation. For example, based on the choice of $\xi_{s}^{u}$ and $\xi_{s}^{v}$, $u(p, s)$ and $v(p, s)$ may or may not be helicity eigenstates. In our calculations we only use the following properties: $\xi_{s}^{u, v \dagger} \xi_{s^{\prime}}^{u, v}=\delta_{s s^{\prime}}$ and $\sum_{s, s^{\prime}} \xi_{s}^{u} \xi_{s^{\prime}}^{v \dagger} \varepsilon_{s s^{\prime}}=-1_{2 \times 2}$. $\sigma^{\mu}=(1, \vec{\sigma})^{\mu}$ and $\bar{\sigma}^{\mu}=(1,-\vec{\sigma})^{\mu}$ in the instant form, where $\vec{\sigma}$ are the usual Pauli matrices.

The general solution to the classical equation of motion can then be written as:

$$
\psi(x)=\int \frac{d p^{+} d^{2} \mathbf{p}}{(2 \pi)^{3} 2 p^{+}}\left(a_{p s} u(p, s) e^{-i p \cdot x}+b_{p s}^{\dagger} v(p, s) e^{i p \cdot x}\right),
$$

where we will soon identify $a_{p s}$ and $b_{p s}^{\dagger}$ as creation and annihilation operators.

In light-cone coordinates, not all the components of $\psi$ are dynamical, meaning that the time derivative of some components do not appear in the Lagrangian. To separate these, one defines projection operators $\Lambda_{+}$and $\Lambda_{-}$as follows.

$$
\Lambda_{ \pm}=\frac{1}{2} \gamma^{\mp} \gamma^{ \pm}=\frac{1}{\sqrt{2}} \gamma^{0} \gamma^{ \pm}=\frac{1}{\sqrt{2}} \gamma^{\mp} \gamma^{0}
$$

They are hermitian matrices satisfying $\Lambda_{+}+\Lambda_{-}=1$, $\Lambda_{ \pm}^{2}=\Lambda_{ \pm}$. One defines the "plus" and "minus" components of $\psi$ as the projections $\psi_{+}=\Lambda_{+} \psi$ and $\psi_{-}=\Lambda_{-} \psi$, respectively. Using the relation $\gamma^{+} \Lambda_{-}=0$ it is easy see that $\psi_{-}$is non-dynamical. Therefore, one can use the equations of motion to write it in terms of the dynamical field $\psi_{+}$, which has the following form:

$\psi_{+}(x)=\int \frac{d p^{+} d^{2} \mathbf{p}}{(2 \pi)^{3} 2 p^{+}}\left(a_{p s} u_{+}(p, s) e^{-i p \cdot x}+b_{p s}^{\dagger} v_{+}(p, s) e^{i p \cdot x}\right)$.

where $u_{+}(p, s)=\Lambda_{+} u(p, s)$ and $v_{+}(p, s)=\Lambda_{+} v(p, s)$. They have norms given by:

$$
u_{+}^{\dagger}(p, s) u_{+}\left(p, s^{\prime}\right)=v_{+}^{\dagger}(p, s) v_{+}\left(p, s^{\prime}\right)=\sqrt{2} p^{+} \delta_{s s^{\prime}} .
$$

The canonical anticommutation relations for the $\psi_{+}$ and $\psi_{+}^{\dagger}$ fields are:

$$
\begin{aligned}
& \left\{\psi_{+}\left(x^{+}, x^{-}, \mathbf{x}\right), \psi_{+}^{\dagger}\left(x^{+}, y^{-}, \mathbf{y}\right)\right\}= \\
& \frac{\Lambda_{+}}{\sqrt{2}} \delta\left(x^{-}-y^{-}\right) \delta^{(2)}(\mathbf{x}-\mathbf{y})
\end{aligned}
$$


The anticommutators of two fields or their hermitian conjugates vanish. Writing $\psi$ in terms of the $a$ and $b$ operators, Eq. (A9), we find that,

$$
\begin{aligned}
& \left\{a_{p s}, a_{q s^{\prime}}^{\dagger}\right\}=\delta_{s s^{\prime}} 2 p^{+}(2 \pi)^{3} \delta\left(p^{+}-q^{+}\right) \delta^{(2)}(\mathbf{p}-\mathbf{q}) \\
& \left\{b_{p s}, b_{q s^{\prime}}^{\dagger}\right\}=\delta_{s s^{\prime}} 2 p^{+}(2 \pi)^{3} \delta\left(p^{+}-q^{+}\right) \delta^{(2)}(\mathbf{p}-\mathbf{q})
\end{aligned}
$$

and that all other anticommutator combinations should be zero. Therefore $a$ and $a^{\dagger}$ can be interpreted as annihilation and creation operators for the fermions, respectively, and $b$ and $b^{\dagger}$ as annihilation and creation operators for the antifermions.

Finally, we describe the quantization of the gauge field. The free field Lagrangian is given by:

$$
\mathcal{L}=-\frac{1}{4} F_{\mu \nu} F^{\mu \nu},
$$

where $F$ is the color field strength tensor. The main new feature that arises during quantization is that we need to fix a gauge. We choose the light-cone gauge, $A^{+}=0$, consistent with the gauge choice for the diagrammatic calculations. In this gauge the operator $A^{-}$is non dynamical and can be eliminated using the equations of motion,

$$
A^{-}=\frac{-1}{\partial_{-}} \partial \cdot \mathbf{A}
$$

The dynamical degrees of freedom are the $\mathbf{A}$ fields. We define two polarization vectors with perpendicular components $\boldsymbol{\epsilon}(+1)=(1 / \sqrt{2})(-1,-i)$ and $\boldsymbol{\epsilon}(-1)=$ $(1 / \sqrt{2})(1,-i)$. The quantized operator $\mathbf{A}$ can then be written as

$$
\mathbf{A}(x)=\int \frac{d p^{+} d^{2} \mathbf{p}}{(2 \pi)^{3} 2 p^{+}}\left(c_{p \lambda} \boldsymbol{\epsilon}(\lambda) e^{-i p \cdot x}+c_{p \lambda}^{\dagger} \boldsymbol{\epsilon}^{*}(\lambda) e^{i p \cdot x}\right) .
$$

with $\lambda= \pm 1$. The canonical commutation relations are,

$$
\begin{aligned}
{\left[\partial_{-} \mathbf{A}^{i}\left(x^{+}, x^{-}, \mathbf{x}\right),\right.} & \left.\mathbf{A}^{j}\left(x^{+}, y^{-}, \mathbf{y}\right)\right]= \\
& \frac{-i \delta^{i, j}}{2} \delta\left(x^{-}-y^{-}\right) \delta^{(2)}(\mathbf{x}-\mathbf{y})
\end{aligned}
$$

which imply:

$$
\left[c_{p \lambda}, c_{q \lambda^{\prime}}^{\dagger}\right]=\delta_{\lambda \lambda^{\prime}} 2 p^{+}(2 \pi)^{3} \delta\left(p^{+}-q^{+}\right) \delta^{(2)}(\mathbf{p}-\mathbf{q})
$$

$c_{p \lambda}$ annihilates as gluon and $c_{p \lambda}^{\dagger}$ creates a gluon. The four-component gauge field $A^{\mu}=\left[A^{+}, A^{-}, \mathbf{A}\right]$ can be written compactly by introducing the four component polarization vectors $\epsilon(p, \pm 1)=\left[0,(\boldsymbol{\epsilon}( \pm 1) \cdot \mathbf{p}) / p^{+}, \boldsymbol{\epsilon}( \pm 1)\right]$, which satisfy $p \cdot \epsilon(p, \lambda)=0$ :

$$
A^{\mu}(x)=\int \frac{d p^{+} d^{2} \mathbf{p}}{(2 \pi)^{3} 2 p^{+}}\left(c_{p \lambda} \epsilon(p, \lambda) e^{-i p \cdot x}+c_{p \lambda}^{\dagger} \epsilon^{*}(p, \lambda) e^{i p \cdot x}\right)
$$

With the creation operators for the particles in hand, we can generate the multi-parton Fock states. A single quark state can be written as:

$$
\left|\vec{p}^{+}, \alpha\right\rangle=a_{\alpha}^{\dagger}\left(\vec{p}^{+}\right)|0\rangle
$$

where the creation operator $a^{\dagger}$ will carry color, spin/helicity/polarization and quark flavor indices $\alpha$, as appropriate. These are normalized as:

$$
\left\langle\vec{q}^{+}, \alpha^{\prime} \mid \vec{p}^{+}, \alpha\right\rangle=\delta_{\alpha \alpha^{\prime}} 2 p^{+}(2 \pi)^{3} \delta^{(3)}\left(\vec{p}^{+}-\vec{q}^{+}\right) .
$$

\section{APPENDIX B: MESON WAVEFUNCTION IN INSTANT AND LIGHT-CONE FORMS}

In this section we describe the relation between the light-cone meson wavefunction and the meson wavefunction in instant form. We do this explicitly for the case of the lowest order Fock component that consists of a heavy quark $Q$ and a light antiquark $\bar{q}$. In the instant form, in the hadron rest frame, we write the meson wavefunction as:

$$
\begin{aligned}
& \left|m_{h}, \overrightarrow{0}\right\rangle_{I}=\sqrt{2 m_{h}} \int \frac{d^{3} \overrightarrow{q_{1}}}{\sqrt{(2 \pi)^{3}}} \frac{d^{3} \overrightarrow{q_{2}}}{\sqrt{(2 \pi)^{3}}} \sqrt{\frac{1}{4 E_{1} E_{2}}} f\left(\overrightarrow{q_{1}}\right) \\
& \quad \times \delta^{(3)}\left(\overrightarrow{q_{1}}+\overrightarrow{q_{2}}\right) \frac{\delta_{c_{1} c_{2}}}{\sqrt{3}} \frac{M_{s_{1} s_{2}}}{\sqrt{2} a_{Q}^{\dagger c_{1} s_{1}}\left(\overrightarrow{q_{1}}\right) b_{q}^{\dagger c_{2} s_{2}}\left(\overrightarrow{q_{2}}\right)|0\rangle,}
\end{aligned}
$$

where $E$ refers to the on-shell energy $E_{i}=\sqrt{\vec{q}_{i}^{2}+m_{i}^{2}}$ and $E_{h}=\sqrt{m_{h}^{2}+\vec{p}^{2}}$ is simply $m_{h}$ in the rest frame. Here, $f(\vec{q})$ represents the momentum space wavefunction normalized such that,

$$
\int \frac{d^{3} \vec{q}}{(2 \pi)^{3}}|f(\vec{q})|^{2}=1 .
$$

In this manuscript we concentrate on distributions $f\left(\vec{q}^{2}\right)$ of spherically-symmetric type, relevant to ${ }^{1} S_{0}$ and ${ }^{3} S_{1}$ open heavy flavor states. The particular example for our case will be a harmonic oscillator distribution $f \sim \exp \left(-\vec{q}^{2} a_{0}^{2} / 2\right)$. The invariant mass squared of the multi-parton system of no net transverse momentum $(\mathbf{P}=0)$ is given by:

$$
M^{2}(\vec{q})=2\left(\sum_{j} p_{i}^{+}\right)\left(\sum_{i} p_{i}^{-}\right)=\sum_{i} \frac{m_{\perp i}^{2}}{x_{i}}
$$

where $m_{\perp i}^{2}=m_{i}^{2}+\mathbf{q}_{i}^{2}$. We see that $M^{2}$ is a function of $\vec{q}$, and the mass of the hadron can be intuitively thought of as $m_{h}^{2}=M^{2}(\langle\vec{q}\rangle)$. For the rest of the section we will concentrate on the specific case of a quark-antiquark state $M^{2}=\left(E_{1}+E_{2}\right)^{2}$. The form of $M^{2}$ simplifies in the following two special cases:

$$
\begin{aligned}
& \vec{q}^{2} \approx \frac{M^{2}}{4}+c_{1}\left(m_{1}, m_{2}\right), \quad \text { if } \vec{q}^{2} \gg m_{i}^{2}, \\
& \vec{q}^{2} \approx \frac{M^{2}}{\frac{\left(m_{1}+m_{2}\right)^{2}}{\left(m_{1} m_{2}\right)}}+c_{2}\left(m_{1}, m_{2}\right), \quad \text { if } \vec{q}^{2} \ll m_{i}^{2} .
\end{aligned}
$$


In all cases the same functional dependence on $\mathbf{k}=\mathbf{q}$ and $x$ is obtained via $\vec{q}^{2} \sim M^{2}$ and the numerical coefficients $c_{i}\left(m_{j}\right)$ are absorbed in the proper normalization of hadron state. This yields:

$$
\psi \sim \exp \left(-\frac{\mathbf{k}^{2}+(1-x) m_{1}^{2}+x m_{2}^{2}}{2 \Lambda^{2} x(1-x)}\right) .
$$

In two limiting cases the coefficient that relates $\Lambda$ in Eq. (B6) to $a_{0}$ (the width of $f\left(\vec{q}^{2}\right)$ ) can be analytically estimated, as discussed above. Since the form of $\psi$ is the same in the two extreme cases, we find it more convenient to treat $\Lambda$ as a constant which can be determined through the requirement that we obtain the same expectation value for the transverse momentum from the instant and light-cone wavefunction forms, i.e. $\left\langle\mathbf{k}^{2}\right\rangle=\left\langle\mathbf{q}^{2}\right\rangle$. For the example of typical open heavy flavor wavefunctions [7] the numerical values are within $30 \%$ of the values for the two limiting cases (B4, B5).

To establish the correspondence between the instantform wavefunction and the light-cone wavefunction, we now convert the spatial momentum integration variables $\vec{q}$ to $\vec{q}^{+}=\left(q^{+}, \mathbf{q}\right)$. The Jacobian for the transformation is $q^{+} / E(\vec{q})$. To recover the (anti)commutation relations outlined in Appendix $\mathrm{A}$ we find that no additional factors arise for the creation and annihilation operators. The proper normalization of states $\propto q^{+}$is ensured when we observe that $\delta\left(q^{z}-q^{z^{\prime}}\right)=\left(q^{+} / E(\vec{q})\right) \delta\left(q^{+}-q^{+^{\prime}}\right)$. Similar relations can be established for antiquarks and gluons. One can also rewrite $\delta\left(q_{1}^{z}+q_{2}^{z}-P^{z}\right) \rightarrow(\sqrt{2}) \delta\left(q_{1}^{+}+\right.$ $\left.q_{2}^{+}-P^{+}\right)$in the boosted frame where we will match the instant and light-cone forms of the state. $P^{+}$is the plus component of the light-cone momentum of the meson. Note that in the meson rest frame $P^{z}=0$. Making these variable changes and recalling that $\mathbf{q}=\mathbf{k}$ leaves us with the same meson state:

$$
\begin{aligned}
& \left|\vec{P}^{+}\right\rangle=\int \prod_{i=1}^{2} \frac{d^{2} \mathbf{k}_{i} d x_{i}}{\sqrt{(2 \pi)^{3}} \sqrt{2 x_{i}}} \delta\left(\sum_{i} x_{i}-1\right) \\
& \times \delta^{(2)}\left(\sum_{i} \mathbf{k}_{i}\right) 2 \sqrt{E_{h}} \sqrt{\frac{E_{1} E_{2}}{q_{1}^{+} q_{2}^{+}}} \frac{M_{s_{1} s_{2}}}{\sqrt{2}} \frac{\delta_{c_{1} c_{2}}}{\sqrt{3}} \\
& \times f\left(\vec{q}^{2}\left(x_{i}, \mathbf{k}_{i}\right)\right) a_{Q}^{\dagger c_{1} s_{1}}\left({\overrightarrow{q_{1}}}^{+}\right) b_{q}^{\dagger c_{2} s_{2}}\left({\overrightarrow{q_{2}}}^{+}\right)|0\rangle .
\end{aligned}
$$

We complete the correspondence by observing:

$$
\begin{aligned}
\lim _{E_{h} \gg m_{h}} 2 \sqrt{E_{h}} \sqrt{\frac{E_{1} E_{2}}{k_{1}^{+} k_{2}^{+}}} f\left(\vec{q}^{2}\left(x_{i}, \mathbf{k}_{i}\right)\right) \\
\rightarrow \sqrt{P^{+} \sqrt{2}} f\left(x_{i}, \mathbf{k}_{i}\right) \equiv \psi\left(x_{i}, \mathbf{k}_{i}\right) .
\end{aligned}
$$

The prefactor of $\sqrt{P^{+}}$in front of the rest frame wavefunction $f(\vec{k})$ in the relation between $\psi\left(x_{i}, \mathbf{k}_{i}\right)$ and $f(\vec{k})$ (Eq. B8) can be physically understood as follows. A boost from the rest frame to a frame with large momentum $P^{+}$leaves the transverse shape of the wavefunction unchanged. However, in the large light-cone momentum direction the wavefunction is stretched in momentum space by a factor $\sqrt{P^{+}}$. Note that Eq. (B8) combined with Eq. (B2) yield $\int d^{2} \mathbf{k} d x|\psi(x, \mathbf{k})|^{2}=2(2 \pi)^{3}$ as employed in this paper.

\section{APPENDIX C: MEDIUM CONTRIBUTION TO HEAVY QUARK FRAGMENTATION FUNCTIONS}

In Sections III and IV we calculated the fragmentation functions for $c$ and $b$ quarks into $D$ and $B$-mesons respectively, from the definition given in [39], Eq. (24). In the adiabatic picture, where the meson wavefunction has sufficient time to equilibrate with the medium, we take the modification of $D$ and $B$ meson wavefunctions in the medium into account by using the thermal wavefunction calculated from potential models in Section IV.

In this equilibrium situation, one can attempt to go a step further by estimating additional corrections that arise from thermal parton pick-up as follows. Instead of taking the expectation value in vacuum, we can define this thermal fragmentation as:

$$
\begin{aligned}
& D_{h / Q}^{T}(z)=z \int \frac{d y^{-}}{2 \pi} e^{i \frac{p^{+}}{z} y^{-}} \frac{1}{3} \operatorname{Tr}_{\text {color }} \frac{1}{2} \operatorname{Tr}_{\text {Dirac }} \frac{\gamma^{+}}{2} \\
\times & \frac{\sum_{m} \exp \left(-\beta E_{m}\right)\left\langle m\left|\psi\left(y^{-}, \mathbf{0}\right) a_{h}^{\dagger}\left(P^{+}\right) a_{h}\left(P^{+}\right) \bar{\psi}(0, \mathbf{0})\right| m\right\rangle}{\sum_{m} \exp \left(-\beta E_{m}\right)} .
\end{aligned}
$$

We take Eq. (C1) to be the minimal generalization of the definition of fragmentation in perturbative QCD, with $z<1$. In Eq. (C1), $E_{m}$ is the energy of the state $|m\rangle$, $\beta$ is the inverse temperature, and $\exp \left(-\beta E_{m}\right)$ gives the thermal weight of the state $m$. For convenience of notation, we are writing the spectrum of states as being discrete, with the states normalized as $\langle m \mid n\rangle=\delta_{m n}$. One can immediately see that in the case that the operator does not contract with the state $m$, we get back the definition in vacuum because the sum of exponentials cancels out between the numerator and the denominator. For weakly interacting quasi-particles, $E_{m}$ is the sum of the energies of the quasi-particles in the state $m$. In this approximation, the denominator

$$
Z=\sum_{m} \exp \left(-\beta E_{m}\right)=\Pi_{\varepsilon_{i}}\left(\exp \left(\beta \varepsilon_{i}\right) \pm 1\right)
$$

where the + is for Fermions and - for the bosons. When the operator contracts with one quasiparticle with energy $\varepsilon$ from the medium (equivalent to saying that the meson picks up a thermal parton of energy $\varepsilon$ ), all the other terms in the product (Eq.C2) cancel out as before, except the one corresponding to energy $\varepsilon$. Hence, one gets an additional factor of $g(\varepsilon)=1 /(\exp (\beta \varepsilon) \pm 1)$ depending on whether the parton is fermion $(+)$ or a boson $(-)$.

At the same order in $\alpha_{s}$ as vacuum fragmentation the following Feynman diagram is the most important additional correction to $D_{h / Q}(z)$ function, Fig.9. We call this correction $D_{h / Q}{ }^{(1)}(z)$. This diagram can be interpreted 


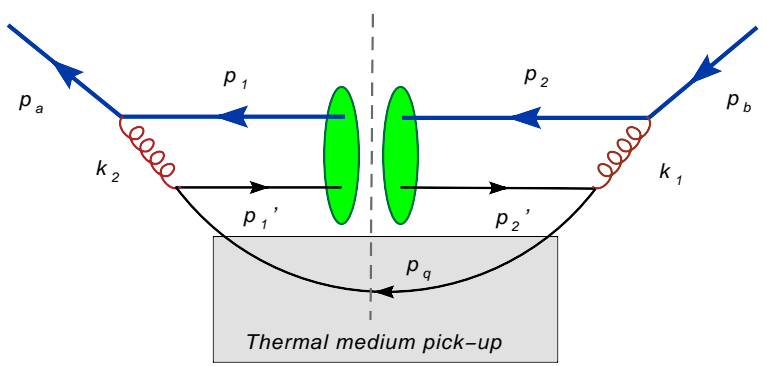

FIG. 9: Thermal correction to the fragmentation function that arises from an in-medium quark pick-up.

as follows. The heavy quark picks up a light thermal antiquark from the medium and forms a meson. The evaluation of this diagram is very similar to Eq. (26) and yields:

$$
\begin{aligned}
& D_{h / Q}^{(1)}(z)=\int \frac{d x_{1} d^{2} \mathbf{k}_{1} \psi\left(x_{1}, \mathbf{k}_{1}\right)}{(2 \pi)^{3} 2 \sqrt{x_{1}\left(1-x_{1}\right)}} \frac{d x_{2} d^{2} \mathbf{k}_{2} \psi^{*}\left(x_{2}, \mathbf{k}_{2}\right)}{(2 \pi)^{3} 2 \sqrt{x_{2}\left(1-x_{2}\right)}} \\
& \frac{M(j)_{s_{1} s_{1}^{\prime}}}{\sqrt{2}} \frac{M(j)_{s_{2} s_{2}^{\prime}}}{\sqrt{2}} \int d s \theta\left(s-\frac{m_{h}^{2}}{z}-\frac{m_{q}^{2}}{1-z}\right) \frac{\alpha_{s}^{2} C_{F}^{2}}{3} \\
& \operatorname{Tr}\left[\gamma^{+} \frac{i}{\gamma \cdot p_{a}-m_{Q}} \gamma^{\mu} u_{s_{1}}\left(p_{1}\right) \bar{v}_{s_{1}^{\prime}}\left(p_{1}^{\prime}\right) \gamma^{\nu}\left(\gamma \cdot p_{q}+m_{q}\right) \gamma^{\sigma}\right. \\
& v_{s_{2}^{\prime}}\left(p_{2}^{\prime}\right) \bar{u}_{s_{2}}\left(p_{2}\right) \gamma^{\lambda} \frac{i}{\gamma \cdot p_{b}-m_{q}} \Pi_{\mu \nu}\left(p_{a}-p_{q}\right) \Pi_{\sigma \lambda}\left(p_{b}-p_{q}\right) \\
& \left.g\left(p_{q}^{0}\right)\right] \frac{1}{\operatorname{Tr}\left[\gamma^{+}(\gamma \cdot p)\right]},
\end{aligned}
$$

where $p_{a}=p_{b}=p_{Q}$ and $p_{q}$ is the momentum of the light antiquark that is picked up from the medium. $g\left(p_{q}^{0}\right)=\frac{1}{e^{\beta p_{q}^{0}+1}}$ is the thermal probability to find a quark with energy $p_{q}^{0}$ in the medium. For future use we define the hadron momentum $p_{h}=p_{1}+p_{1}^{\prime}=p_{2}+p_{2}^{\prime}$. The expression, Eq. (C3), differs from $D_{h / Q}(z)$ only in the extra factor $g\left(p_{q}^{0}\right)$. To get a rough estimate of the correction, we replace $p_{q}^{0}$ by its typical value $\bar{p}_{q}^{0}$ and obtain,

$$
D^{(1)}(z) \sim D(z) g\left(\bar{p}_{q}^{0}\right) .
$$

¿From the Feynman diagram Fig. (9) we see that $p_{q}=$ $p_{a}-p_{h}$. For a typical sample of the kinematics, we consider the following. By choosing an appropriate direction of the axes, the transverse momentum of the hadron can be taken to be zero. Then,

$$
\begin{aligned}
& p_{a}=\left[\frac{P^{+}}{z}, p_{a}^{-}, \mathbf{p}_{q}\right] \\
& p_{h}=\left[P^{+}, \frac{m_{h}^{2}}{2 z p^{+}}, \mathbf{0}\right] \\
& p_{\bar{q}}=\left[(1 / z-1) P^{+}, \frac{\mathbf{p}_{q}^{2}+m_{q}^{2}}{2 P^{+}(1 / z-1)}, \mathbf{p}_{q}\right]
\end{aligned}
$$

where $\mathbf{p}_{q}$ is the transverse momentum of the light antiquark and is related to the off-shellness $\left(p_{a}^{2}-m_{Q}^{2}\right)$ of the initial heavy quark. We obtain straightforwardly:

$$
\begin{aligned}
\bar{p}_{q}^{0} & =\frac{1}{\sqrt{2}}\left[P^{+}\left(\frac{1}{z}-1\right)+\frac{m_{q}^{2}}{2 P^{+}(1 / z-1)}\right] \\
& \geq \frac{1}{\sqrt{2}} P^{+}(1 / z-1),
\end{aligned}
$$

where $m_{q}$ is the light quark mass in the thermal medium. To obtain a conservative estimate for the correction, we take $m_{q}$ to be zero. Using typical value of $z$ for $D$ and $B$-mesons, we find that for a $4 \mathrm{GeV} D$-meson moving through the medium at a temperature $250 \mathrm{MeV}$, this gives $g\left(\bar{p}_{q}^{0}\right)$ less than $3 \%$, while for a $4 \mathrm{GeV} B$-meson moving through the same medium we find corrections less than $15 \%$.

\section{APPENDIX D: ANALYTIC MODEL OF HEAVY QUARK AND HEAVY MESON MULTIPLICITIES}

It is useful to find an approximate analytic solution to the system of ordinary differential equations, Eqs. (47) and (48), for the purpose of understanding the interplay between heavy quark fragmentation and meson dissociation and evaluating the fraction of the time during which the charm is in a partonic state. This will help us determine the quenched initial conditions for our numerical results. We will take the $p_{T}$-dependent quark and hadron multiplicities, $f^{Q}\left(p_{T}, t\right)=A_{Q} / p_{T}^{n_{Q}}$ and $f^{H}\left(p_{T}, t\right)=A_{H} / p_{T}^{n_{H}}$ to be of power-law type [28]. We can then express:

$$
\int_{0}^{1} d x \frac{1}{x^{2}} \phi_{Q / H}(x) f^{H}\left(p_{T} / x\right)=f^{H}\left(p_{T} / c_{H}\right),
$$

and

$$
\int_{0}^{1} d z \frac{1}{z^{2}} D_{H / Q}(z) f^{Q}\left(p_{T} / z\right)=f^{Q}\left(p_{T} / c_{Q}\right) .
$$

For any finite $p_{T}$ range the coefficients $n_{H}, n_{Q}, c_{H}, c_{Q}$ can be determined numerically. In order to obtain a simple analytic solution we must also average the formation and dissociation rates in the interval $\left(0, L_{Q G P}\right)$. This yields:

$$
\begin{aligned}
\partial_{t} f^{Q}\left(p_{T}, t\right) & =-\frac{f^{Q}\left(p_{T}, t\right)}{\left\langle\tau_{\text {form }}\left(p_{T}\right)\right\rangle}+\frac{c_{H}^{n_{H}} f^{H}\left(p_{T}, t\right)}{\left\langle\tau_{\text {diss }}\left(p_{T}\right)\right\rangle}, \\
\partial_{t} f^{H}\left(p_{T}, t\right) & =-\frac{f^{H}\left(p_{T}, t\right)}{\left\langle\tau_{\text {diss }}\left(p_{T}\right)\right\rangle}+\frac{c_{Q}^{n_{Q}} f^{Q}\left(p_{T}, t\right)}{\left\langle\tau_{\text {form }}\left(p_{T}\right)\right\rangle} .
\end{aligned}
$$

A general solution ansatz $\propto \sum A_{i} e^{r_{i} t}$ leads to:

$$
\begin{aligned}
& r_{1,2}=\frac{1}{2}\left[-\left(\frac{1}{\left\langle\tau_{\text {form }}\right\rangle}+\frac{1}{\left\langle\tau_{\text {diss }}\right\rangle}\right)\right. \\
& \left. \pm \sqrt{\left(\frac{1}{\left\langle\tau_{\text {form }}\right\rangle}+\frac{1}{\left\langle\tau_{\text {diss }}\right\rangle}\right)^{2}-\frac{4}{\tau_{\text {form }} \tau_{\text {diss }}}\left(1-c_{Q}^{n_{Q}} c_{H}^{n_{H}}\right)}\right]
\end{aligned}
$$


rvalues From the initial conditions, Eq. (49), and Eqs. (D1) and (D2) we can also determine the time derivatives of the heavy quark and hadron distributions at $t=0$. Solving for the coefficients in the linear superposition of independent solutions we find:

$$
\begin{aligned}
f^{H}\left(p_{T}, t\right)= & {\left[\frac{c_{Q}^{n_{Q}} e^{r_{1} t}}{\left\langle\tau_{\text {form }}\left(p_{T}\right)\right\rangle\left(r_{1}-r_{2}\right)}\right.} \\
& \left.-\frac{c_{Q}^{n_{Q}} e^{r_{2} t}}{\left\langle\tau_{\text {form }}\left(p_{T}\right)\right\rangle\left(r_{1}-r_{2}\right)}\right] f^{Q}\left(p_{T}, 0\right),
\end{aligned}
$$

and,

$$
\begin{aligned}
f^{Q}\left(p_{T}, t\right)= & -\left[\frac{e^{r_{1} t}}{r_{1}-r_{2}}\left(\frac{1}{\left\langle\tau_{\text {form }}\left(p_{T}\right)\right\rangle}+r_{2}\right)\right. \\
& \left.-\frac{e^{r_{2} t}}{r_{1}-r_{2}}\left(\frac{1}{\left\langle\tau_{\text {form }}\left(p_{T}\right)\right\rangle}+r_{1}\right)\right] f^{Q}\left(p_{T}, 0\right) .
\end{aligned}
$$

We can use Eqs. (D4) and (D5) not only to study the mix of heavy quarks and hadrons as a function of $\left\langle\tau_{\text {form }}\left(p_{T}\right)\right\rangle,\left\langle\tau_{\text {diss }}\left(p_{T}\right)\right\rangle$ but to also evaluate the fraction of the time the charm or bottom are in a partonic state:

$$
\frac{t_{\text {partonic }}\left(p_{T}\right)}{t_{\text {total }}}=\frac{\int_{0}^{L_{Q G P}} d t t f^{Q}\left(p_{T}, t\right)}{\int_{0}^{L_{Q G P}} d t t f^{Q}\left(p_{T}, t\right)+\int_{0}^{L_{Q G P}} d t t f^{H}\left(p_{T}, t\right)} .
$$

[1] A. D. Frawley, T. Ullrich and R. Vogt, Phys. Rept. 462, 125 (2008); references therein.

[2] D. d'Enterria, arXiv:0902.2011 [nucl-ex].

[3] G. David, arXiv:0903.0336 [nucl-ex].

[4] M. Gyulassy, I. Vitev, X. N. Wang and B. W. Zhang, arXiv:nucl-th/0302077

[5] B. W. Zhang, E. Wang and X. N. Wang, Phys. Rev. Lett. 93, 072301 (2004).

[6] S. Wicks, W. Horowitz, M. Djordjevic and M. Gyulassy, Nucl. Phys. A 783, 493 (2007).

[7] A. Adil and I. Vitev, Phys. Lett. B 649, 139 (2007).

[8] F. Dominguez and B. Wu, Nucl. Phys. A 818, 246 (2009).

[9] F. Dominguez, C. Marquet, and B. Wu, Nucl. Phys. A 823, 99 (2009).

[10] M. Burkardt, Adv. Nucl. Phys. 23, 1 (1996)

[11] S. J. Brodsky, H. C. Pauli and S. S. Pinsky, Phys. Rept. 301, 299 (1998).

[12] M. Avila, Phys. Rev. D 49, 309 (1994).

[13] M. A. Avila, Mod. Phys. Lett. A 142059 (1999).

[14] A. Mocsy and P. Petreczky, Phys. Rev. D 77, 014501 (2008).

[15] O. Kaczmarek and F. Zantow, Phys. Rev. D 71, 114510 (2005).

[16] A. Adare et al. [PHENIX Collaboration], Phys. Rev. Lett. 98, 172301 (2007).

[17] B. I. Abelev et al. [STAR Collaboration], Phys. Rev. Lett. 98, $192301(2007)$

[18] I. Vitev, T. Goldman, M. B. Johnson and J. W. Qiu, Phys. Rev. D 74, 054010 (2006).

[19] V. N. Gribov, Sov. Phys. JETP 29, 483 (1969) [Zh. Eksp. Teor. Fiz. 56, 892 (1969)].

[20] F. I. Olness, R. J. Scalise and W. K. Tung, Phys. Rev. D 59, 014506 (1999).

[21] J. F. Owens, Rev. Mod. Phys. 59, 465 (1987).
[22] A. Accardi, arXiv:hep-ph/0212148, references therein.

[23] I. Vitev, Phys. Lett. B 562, 36 (2003).

[24] I. Vitev, Phys. Rev. C 75, 064906 (2007).

[25] J. W. Qiu and G. Sterman, Int. J. Mod. Phys. E 12, 149 (2003).

[26] J. W. Qiu and I. Vitev, Phys. Rev. Lett. 93, 262301 (2004).

[27] J. W. Qiu and I. Vitev, Phys. Lett. B 632, 507 (2006).

[28] I. Vitev and B. W. Zhang, Phys. Lett. B 669, 337 (2008).

[29] T. Hirano, N. van der Kolk and A. Bilandzic, arXiv:0808.2684 [nucl-th]; references therein.

[30] A. Dumitru, Y. Nara and M. Strickland, Phys. Rev. D 75, 025016 (2007)

[31] B. B. Back et al. [PHOBOS Collaboration], Phys. Rev. Lett. 88, 022302 (2001).

[32] I. Vitev, S. Wicks and B. W. Zhang, JHEP 0811, 093 (2008)

[33] A. Adare et al. [PHENIX Collaboration], Phys. Rev. Lett. 101, 162301 (2008).

[34] A. Adare et al. [PHENIX Collaboration], Phys. Rev. C 77, 064907 (2008)

[35] B. I. Abelev et al. [STAR Collaboration], Phys. Lett. B 655, 104 (2007).

[36] G. Wang [STAR Collaboration], J. Phys. G: Nucl. Part. Phys. 35104107 (2008).

[37] J. P. Ma, Phys. Lett. B 332, 398 (1994).

[38] B. Q. Ma, Z. Phys. A 345, 321 (1993).

[39] J. C. Collins and D. E. Soper, Nucl. Phys. B 194, 445 (1982).

[40] C. H. Chang and Y. Q. Chen, Phys. Lett. B 284, 127 (1992).

[41] E. Braaten, K. M. Cheung, S. Fleming and T. C. Yuan, Phys. Rev. D 51, 4819 (1995).

[42] R. Mertig, M. Bohm and A. Denner, Comput. Phys. 
Commun. 64, 345 (1991).

[43] N. Brambilla, A. Pineda, J. Soto and A. Vairo, Rev. Mod. Phys. 77, 1423 (2005).

[44] A. Mocsy and P. Petreczky, Phys. Rev. Lett. 99, 211602 (2007).

[45] J. J. Sakurai, Advanced Quantum Mechanics, First ISE reprint, Addison-Wesley Publising Company Inc. (1999)
[46] W. Greiner, Relativistic Quantum Mechanics, Berlin, Springer-Verlag (1990).

[47] W. H. Press et al., Numerical Recipes in Fortran, New York, Cambridge University Press (1989).

[48] T. Sjostrand, S. Mrenna and P. Skands, JHEP 0605, 026 (2006). 